\title{
INFINITE SYSTEMS OF ORDINARY DIFFERENTIAL EQUATIONS WITH APPLICATIONS TO CERTAIN SECOND-ORDER PARTIAL DIFFERENTIAL EQUATIONS*
}

BY

DANIEL C. LEWIS, JR.

\section{INTRODUCTION}

From a purely formal point of view, the problem of integrating the nonlinear partial differential equation

$$
\frac{\partial^{2} u}{\partial t^{2}}-\frac{\partial^{2} u}{\partial y^{2}}=F\left(\frac{\partial u}{\partial y}, \frac{\partial u}{\partial t}, u, y, t\right)
$$

under the conditions $u(0, t)=u(\pi, t)=0, u(y, 0)=f(y), u_{t}(y, 0)=g(y)$ (where $f$ and $g$ are prescribed functions) can be reduced in the following way to the problem of integrating an infinite system of ordinary differential equations,

$$
\frac{d^{2} x_{n}}{d t^{2}}+n^{2} x_{n}=f_{n}\left[t, x_{1}, \frac{d x_{1}}{d t}, x_{2}, \frac{d x_{2}}{d t}, \ldots\right] \quad(n=1,2,3, \cdots) .
$$

We want the solution to be valid in a rectangular region, $0 \leqq y \leqq \pi$, $0 \leqq t \leqq K>0$. We assume the trigonometric developments

$$
f(y)=\sum_{k=1}^{\infty} a_{k} \sin k y, g(y)=\sum_{k=1}^{\infty} a_{k}^{\prime} \sin k y, u(y, t)=\sum_{k=1}^{\infty} x_{k}(t) \sin k y,
$$

where the $a_{k}$ and $a_{k}^{\prime}$ are known constants and the $x_{k}(t)$ are unknown functions. If we formally differentiate the series for $u(y, t)$, substitute in the partial differential equation, multiply through by $(2 / \pi) \sin n y$, and integrate with respect to $y$ from 0 to $\pi$, making use of the orthogonal properties of the sine functions, we get the $n$th equation of the infinite system written above with

$$
\begin{aligned}
f_{n}[t, & \left.x_{1}, \frac{d x_{1}}{d t}, x_{2}, \frac{d x_{2}}{d t}, \ldots\right] \\
& =\frac{2}{\pi} \int_{0}^{\pi} F\left[\sum_{k=1}^{\infty} x_{k} k \cos k y, \sum_{k=1}^{\infty} \frac{d x_{k}}{d t} \sin k y, \sum_{k=1}^{\infty} x_{k} \sin k y, y, t\right] \sin n y d y .
\end{aligned}
$$

- Presented to the Society, December 27, 1932; received by the editors September 20, 1932, and in revised form and with addition of Part III, Feburary 7, 1933. 
We must evidently solve our infinite system under the initial conditions $x_{k}(0)=a_{k}, d x_{k} /\left.d t\right|_{t=0}=a_{k}^{\prime}$.

It is the principal object of this paper to put the above formal procedure upon a rigorous basis.

In Part I, we shall study the slightly more general system

$$
\frac{d^{2} x_{n}}{d t^{2}}+\mu_{n}^{2} x_{n}=f_{n}\left[t, x_{1}, \frac{d x_{1}}{d t}, x_{2}, \frac{d x_{2}}{d t}, \ldots\right] \quad(n=1,2, \cdots),
$$

the $\mu_{n}$ being arbitrary positive constants, together with the initial conditions given above. Actually we shall study this system in the equivalent integral form,

$$
\begin{aligned}
x_{n}(t)= & a_{n} \cos \mu_{n} t+\left(a_{n}^{\prime} / \mu_{n}\right) \sin \mu_{n} t \\
& +\left(1 / \mu_{n}\right) \int_{0}^{t} f_{n}\left[\tau, x_{1}(\tau), \frac{d x_{1}}{d t}(\tau), \cdots\right] \sin \mu_{n}(t-\tau) d \tau .
\end{aligned}
$$

In Part II, we shall apply the results of Part I to partial differential equations, thus obtaining an existence theorem.

This plan has already been carried out by L. Lichtenstein* for equations of considerably more restricted type. The right hand side of Lichtenstein's equation is, in fact, independent of $\partial u / \partial y$ and $\partial u / \partial t$ and can be developed in a power series in $u$ :

$$
F\left(\frac{\partial u}{\partial y}, \frac{\partial u}{\partial t}, u, y, t\right)=\sum_{k=1}^{\infty} p_{k}(y, t) u^{k} .
$$

On the other hand, the essential requirement laid down by us is that $F$ should obey a certain Lipschitz condition in its first three arguments. The present results also represent a generalization beyond Lichtenstein's work in that the requirements on the initial values, $f(y)$ and $g(y)$, are much less restrictive. Here it is merely assumed that $f^{\prime}(y)$ and $g(y)$ have summable squares on $0 \leqq y \leqq \pi$, or in other words that $\sum k^{2} a_{k}{ }^{2}$ and $\sum a_{k}{ }^{2}$ converge; whereas Lichtenstein assumes the convergence of $\sum k^{2}\left|a_{k}\right|$ and $\sum k\left|a_{k}{ }^{\prime}\right|$. The generalizations that Lichtenstein does carry through in other directions (as to the shape of the region and the nature of the end or "boundary" conditions) can equally well be carried out here.

On the other hand our generalizations are gained at a certain sacrifice. The solution $u(y, t)$ produced by Lichtenstein is a solution in the ordinary sense, whereas the $u(y, t)$ produced by us may be a solution only in a certain generalized sense to be defined later. This generalized notion of a solution of a partial differential equation is, however, a natural one, and has been used

* See bibliography at the end of this introduction. 
by other authors. N. Wiener, ${ }^{*}$ for example, has given a generalization, which, while not assuming the existence of the first derivatives, $\partial u / \partial y$ and $\partial u / \partial t$, applies only to linear equations. He gives references to Bôcher and G. C. Evans. My own definition requires the existence of the first derivatives, but, so far as I know, it is the only one which applies to the general second-order partial differential equation, linear or not.

A bibliography of the literature on infinite systems of differential equations appears at the end of this introduction. This bibliography is complete so far as I have been able to ascertain. None of the work there listed, with the exception of Lichtenstein's and Siddiqi's, can be applied here. The reason is that the usual existence theorems for infinite systems of differential equations of the form $d z_{k} / d t=\zeta_{k}\left(t, z_{1}, z_{2}, \cdots\right)$ with initial conditions $z_{k}(0)=c_{k}$, assume a too restrictive correspondence between the laws of decrease of the $\left|z_{k}-c_{k}\right|$ and the $\left|\zeta_{k}\right|$. This correspondence is roughly of the nature that the convergence of $\sum_{k}\left|z_{k}-c_{k}\right|^{2}$ implies the convergence of $\sum_{k}\left|\zeta_{k}\right|^{2}$ for $t$ suitably restricted. Evidently such an assumption fails to take into account even the following highly degenerate example which can be integrated immediately:

$$
\begin{array}{rr}
\frac{d z_{2 n-1}}{d t}=n z_{2 n} \equiv \zeta_{2 n-1}\left(t, z_{1}, z_{2}, \cdots\right), & z_{k}(0)=c_{k}, \\
\frac{d z_{2 n}}{d t}=-n z_{2 n-1} \equiv \zeta_{2 n}\left(t, z_{1}, z_{2}, \cdots\right) & (n, k=1,2,3, \cdots) .
\end{array}
$$

Assume the convergence of $\sum_{k} c_{k}^{2}$. Then the convergence of $\sum_{k} z_{k}^{2}$ would ensure the convergence of $\sum_{k}\left|z_{k}-c_{k}\right|^{2}$, but not that of $\sum_{k} \zeta_{k}^{2}$. Nevertheless such an infinite system is extremely useful in the applications to partial differential equations. This particular simple system is included in the theories presented both by Lichtenstein and by me. For it may be written in the form

if we set $x_{n}=z_{2 n-1}$,

$$
\frac{d^{2} x_{n}}{d t^{2}}+n^{2} x_{n}=0
$$

$$
\frac{d x_{n}}{d t}=n z_{2 n}
$$

But it can be easily shown that a large field still awaits exploration.

The infinite systems considered in this paper are formally quite like those treated by Lichtenstein and quite unlike those treated by W. L. Hart in his paper of 1922. Nevertheless the methods are much more similar to Hart's

* Mathematische Annalen, vol. 95 (1926), p. 582. 
methods than to Lichtenstein's; and the author wishes to acknowledge here his less obvious debt to Hart.

The application of the results of Part I are probably not limited to the problems considered in Part II. Instead of using the trigonometric expansions, exclusively considered in Part II, one might use general Sturm-Liouville orthogonal functions. Such a procedure might furnish theories for non-linear normal hyperbolic equations (in any number of independent variables) with boundary conditions of a much more complicated type than those considered here. Here also is a large field awaiting exploration.

Existence theorems for the Cauchy problem with non-analytic initial conditions have not yet been given for general non-linear* hyperbolic equations, except for the case of two independent variables, which has been most elegantly treated by $\mathrm{H}$. Lewy. $\dagger$ It may be that the method of infinite systems of ordinary differential equations will furnish the key to the problem. Even in the case of two independent variables Lewy's work is applicable only to the unmixed Cauchy problem, whereas this method is applicable to the mixed problem, where boundary conditions as well as initial conditions play a prominent rôle. Further developments await more general existence theorems for infinite systems of differential equations.

\section{BIBLIOGRAPHY OF THE THEORY OF INFINITE SYSTEMS OF DIFFERENTIAL} EQUATIONS

H. von Koch, Sur les systèmes d'ordre infini d'équations différentielles, Öfversigt af Kongliga Vetenskaps-Akademiens Förhandlingar, vol. 56 (1899), pp. 395-411 (analytic non-linear theory).

E. H. Moore, New Haven Mathematical Colloquium, 1906 (a linear theory in the sense of "general analysis").

F. R. Moulton, Solution of an infinite system of differential equations of the analytic type, Proceedings of the National Academy of Sciences, vol. 1 (1915), pp. 350-354 (analytic non-linear theory). The same work is published in the text-book on differential equations by the same author.

T. H. Hildebrandt, On a theory of linear differential equations in general analysis, these Transactions, vol. 18 (1917), pp. 73-96.

W. L. Hart, Differential equations and implicit functions in infinitely many

* Riemann and Hadamard have laid the foundation for the linear case. See the latter's book Lectures on Cauchy's Problem.

See also M. Mathisson, Eine neue Lösungsmethode für Differentialgleichungen von normalen hyperbolischen Typus, Mathematische Annalen, vol. 107 (1932), pp. 400-419.

$\dagger \ddot{U}$ ber das Anfangswertproblem einer hyperbolischen nichtlinearen partiellen Differentialgleichung zweiter Ordnung mit zwei unabhängigen Veränderlichen, Mathematische Annalen, vol. 98 (1927), pp. 179-191. 
variables, these Transactions, vol. 18 (1917), pp. 125-160; Functions of infinitely many variables in Hilbert space, these Transactions, vol. 23 (1922), pp. 30-50 (non-analytic non-linear theory); Linear differential equations in infinitely many variables, American Journal of Mathematics, vol. 39 (1917), pp. 407-424; The Cauchy-Lipschitz method for infinite systems of differential equations, American Journal of Mathematics, vol. 43 (1921), pp. 226-231.

I. A. Barnett, Differential equations with a continuous infinitude of variables, American Journal of Mathematics, vol. 44 (1922), pp. 172-190; Linear partial differential equations with a continuous infinitude of variables, American Journal of Mathematics, vol. 45 (1923), pp. 42-53.

A. Wintner, Zur Theorie der unendlichen Differentialsysteme, Mathematische Annalen, vol. 95 (1925), pp. 544-556; Zur Lösung von Differentialsystemen mit unendlich vielen Veränderlichen, Mathematische Annalen, vol. 98 (1927), pp. 273-280 (analytic non-linear theory); Zur Analysis im Hilbertschen Raume, Mathematische Zeitschrift, vol. 28 (1928), pp. 451-470; Upon $a$ theory of infinite systems of non-linear implicit and differential equations, American Journal of Mathematics, vol. 53 (1931), pp. 241-257.

L. Lichtenstein, Zur Theorie partieller Differentialgleichungen zweiter Ordnung vom hyperbolischen Typus, Journal für die reine und angewandte Mathematik, vol. 158 (1927), pp. 80-91.

W. T. Reid, Properties of solutions of an infinite system of ordinary linear differential equations of the first order with auxiliary boundary conditions, these Transactions, vol. 32 (1930), pp. 284-318.

M. R. Siddiqi, Zur Theorie der nichtlinearen partiellen Differentialgleichungen vom parabolischen Typus, Mathematische Zeitschrift, vol. 35 (1932), pp. 464-484.

In addition to the above papers there is also an extensive literature dealing with the single differential equation of infinite order in one unknown function. This theory is closely connected with the Heaviside operational calculus and has little or nothing in common with the theory of infinite systems of differential equations. The device whereby the differential equation

$$
F\left(t, x, \frac{d x}{d t}, \frac{d^{2} x}{d t^{2}}, \ldots, \frac{d^{n} x}{d t^{n}}\right)=0
$$

can be put into the form of a system of equations

$$
\frac{d x_{k}}{d t}=f_{k}\left(t, x_{1}, x_{2}, \cdots, x_{n}\right) \quad(k=1,2, \cdots, n),
$$

by setting

$$
x_{k}=\frac{d^{k-1} x}{d t^{k-1}},
$$


apparently fails when $n=\infty$. The bibliography of the single equation of infinite order may be found in a footnote to a paper by H. T. Davis in the Annals of Mathematics, (2), vol. 32 (1931), pp. 686-714. It mentions the following authors: Bourlet, Bromwich, von Koch, Pincherle, Ritt, Schürer, Scheffer, Valiron, Wiener.

\section{PART I}

1. Notation, terminology, definitions, and lemmas. We consider infinite systems of equations of the form,

$$
x_{k}(t)=\phi_{k}(t)+\frac{1}{\mu_{k}} \int_{0}^{t} f_{k}\{\tau, x(\tau)\} \sin \mu_{k}(t-\tau) d \tau(k=1,2, \cdots) .
$$

The $x_{k}(t)$ are the unknown functions.

The $\mu_{k}$ are any positive numbers.

$\phi_{k}(t)$ is an abbreviation for $a_{k} \cos \mu_{k} t+\left(a_{k}^{\prime} / \mu_{k}\right) \sin \mu_{k} t$, where $a_{k}$ and $a_{k}{ }^{\prime}$ are for the present completely arbitrary, except that, in common with all other numbers arising in this paper, they are real.

$f_{k}\{\tau, x(\tau)\}$ is a function depending upon $k, \tau, x_{1}(\tau),(d / d \tau) x_{1}(\tau), x_{2}(\tau)$, $(d / d \tau) x_{2}(\tau), \cdots$.

In general, an italic letter followed by $\{t, x\}$ will be an abbreviation for a function dependent upon the infinitely many independent variables, $t, x_{1}$, $x_{1}^{\prime}, x_{2}, x_{2}^{\prime}, x_{3}, x_{3}^{\prime}, \cdots$. On the other hand, a Greek letter, with a superscript $n$, followed by $\{t, x\}$ will indicate a function of the first $2 n+1$ of these variables

Thus $F\{t, x\}$ depends upon $t, x_{1}, x_{1}^{\prime}, x_{2}, x_{2}^{\prime}, \cdots$, while $\psi^{(n)}\{t, x\}$ depends upon $t, x_{1}, x_{1}^{\prime}, \cdots, x_{n}, x_{n}^{\prime}$ only.

By a "point" in "function space" we shall mean an infinite sequence of numbers, called "coordinates." We shall deal with two types of function space:

In considering type 1 , the $n$th coordinate of a point will usually be denoted by a letter with the subscript $n$, e.g. $x_{n}$. A point in function space of type 1 , whose coordinates are represented by $x_{1}, x_{2}, x_{3}, \cdots$, will be denoted briefly by $[x]$.

In dealing with type 2 , the $n$th coordinate will be denoted by a letter unprimed with the subscript $\frac{1}{2}(n+1)$, if $n$ is odd, and primed with the subscript $\frac{1}{2} n$, if $n$ is even. Thus the symbols $x_{1}, x_{1}^{\prime}, x_{2}, x_{2}^{\prime}, x_{3}, x_{3}^{\prime}, \cdots$ may be taken to represent in the proper order the coordinates of a point in space of type 2 . Such a point with coordinates represented by these symbols is denoted by $(x)$. Here we use parentheses instead of the square brackets reserved for points of function space of type 1 . 
We shall use different "distance" functions for the two spaces. We begin by defining the following symbols:

$$
\begin{aligned}
|x|_{m, n} & =\left[\sum_{k=m}^{n}\left|x_{k}\right|^{p}\right]^{1 / p} ;|x|=|x|_{1, \infty} \text { if this limit exists; } \\
\|x\|_{m, n} & =\left[\sum_{k=m}^{n} \mu_{k}^{p}\left|x_{k}\right|^{p}+\sum_{k=m}^{n}\left|x_{k}^{\prime}\right|^{p}\right]^{1 / p} ;\|x\|=\|x\|_{1, \infty} \text { if this limit exists. }
\end{aligned}
$$

$p$ is a positive constant not less than 1 . For the sake of brevity, the dependence of these symbols on $\mu_{k}$ and $p$ is not indicated. The "distance" between two points $[b]$ and $[c]$ is defined as $|b-c|$. The "distance" between two points $(b)$ and $(c)$ is defined as $\|b-c\|$.

The symbols obey the following classic inequalities:

$$
\begin{array}{rlrl}
\mid b+d_{m, n} & \leqq|b|_{m, n}+|c|_{m, n} ; & |b+d| \leqq|b|+|d| ; \\
\|b+c\|_{m, n} & \leqq\|b\|_{m, n}+\|c\|_{m, n} ; \quad\|b+c\| \leqq\|b\|+\|c\| .
\end{array}
$$

For our purposes, a region in function space is simply the collection of points whose coordinates satisfy certain conditions. These conditions are usually given in the form of inequalities. Two very special regions $Q$ and $R$ will be largely used in this paper. They are defined as follows:

Let $q$ be a positive number. The point $(x)$ belongs to the region $Q(q)$ if

$$
\|x\| \leqq q .
$$

Let $r$ be a positive number. The point (x) belongs to the region $R(r)$ if for at least one value of $t$ the inequality

$$
\|x-\phi(t)\| \leqq r \text { is valid, }
$$

where $\phi_{1}(t), \phi_{2}(t), \cdots$ have already been defined and $\phi_{k}{ }^{\prime}(t)=(d / d t) \phi_{k}(t)$.

The functions $f_{k}\{t, x\}$ which we consider are of a special type which we shall call "convergent." A function of this type, depending upon an infinite number of variables, is defined as the limit of a sequence of functions, each one of which depends only upon a finite number of variables. To be more precise, we write a formal definition:

Definition. A function $f\{t, x\}$, defined for $t$ in some interval, $0 \leqq t \leqq T$, and for $(x)$ in some region $S$ of (type 2) function space, is said to be of convergent type, if there exists a sequence of functions, $\psi^{(n)}\{t, x\}, n=1,2, \cdots$, the nth function $\psi^{(n)}\{t, x\}$ being defined for $0 \leqq t \leqq T$ and for all sets of values for $x_{1}$,

* Cf. F. Riesz, Les Systèmes d' Equations Lineaires d une Infinité d'Inconnues, p. 43 et seq. 
$x_{1}^{\prime}, x_{2}, x_{2}^{\prime}, \cdots, x_{n}, x_{n}^{\prime}$ which are the first $2 n$ coordinates of any point $(x)$ in $S$, such that for any fixed point $(x)$ in $S$,

$$
\lim _{n=\infty} \psi^{(n)}\{t, x\}=f\{t, x\} \text {. }
$$

The usefulness of this definition rests on the following lemma:

LEMMA 1. Let $\psi^{(n)}$ be continuous in its $2 n+1$ arguments. Let $\left|\psi^{(n)}\{t, x\}\right|$ $\leqq M$, where $M$ is some number independent of $n, t, x_{1}, x_{1}^{\prime}, x_{2}, x_{2}^{\prime}, \cdots$. Let $x_{1}(t), x_{2}(t), \cdots$ be a set of functions, each of which is defined and of class $C^{\prime}$ on $0 \leqq t \leqq T$, and set $x_{k}^{\prime}(t)=(d / d t) x_{k}(t)$. Let these functions be such that $(x(t))$ lies in $S$ for $0 \leqq t \leqq T$. Finally let $g(t)$ be defined and integrable on $0 \leqq t \leqq T$.

Then $\int_{0}^{T} f\{t, x(t)\} g(t) d t$ exists in the sense of Lebesgue and is in fact equal to $\lim _{n=\infty} \int_{0}^{T} \psi^{(n)}\{t, x(t)\} g(t) d t$.

The proof of this lemma together with the following corollary is left to the reader.

CoRollary. Let $\mu$ be a constant. Then under the hypotheses of Lemma 1 $\int_{0}^{t} f\{\tau, x(\tau)\} \sin \mu(t-\tau) d \tau$ is of class $C^{\prime}$ for $0 \leqq t \leqq T$, possessing almost everywhere in this interval a second derivative.

We need two more simple inequalities before proceeding to the existence proof of the next section.

If $f(t)$ is integrable on $0 \leqq t \leqq T$, we have the classic inequality

$$
\left[\int_{0}^{t}|f(\tau)| d \tau\right]^{p} \leqq t^{p-1} \int_{0}^{t}|f(\tau)|^{p} d \tau^{*} \text { for } 0 \leqq t \leqq T .
$$

Hence, if $f_{1}(t), f_{2}(t), \cdots$ is an infinite sequence of functions, each of which is integrable on $0 \leqq t \leqq T$, then

$$
\left|\int_{0}^{t}\right| f(\tau)|d \tau|_{m, n}^{p} \leqq t^{p-1} \int_{0}^{t}|f(\tau)|_{m, n}^{p} d \tau \text { for } 0 \leqq t \leqq T .
$$

If $|f(t)|_{m, n}$ is bounded uniformly with respect to $n$ and $t$, and if $|f(t)|_{m, \infty}$ exists for each $t$, we can, by Lebesgue's theorem, pass to the limit and write

$$
\left|\int_{0}^{t}\right| f(\tau)|d \tau|_{m, \infty}^{p} \leqq t^{p-1} \int_{0}^{t}|f(\tau)|_{m, \infty}^{p} d \tau .
$$

2. Fundamental existence theorem for equations (1.1). We suppose that there exist four positive numbers $r, A, B, T$, such that the following three hypotheses hold:

* E. W. Hobson, Functions of a Real Variable, 3d edition, vol. 1, p. 643. 
HYPOTHESIS 2.I. The $f_{k}\{t, x\}$ are defined and are of convergent type for (x) in $R(r)$ and for $0 \leqq t \leqq T$. The approximation functions $\psi_{k}^{(n)}\{t, x\}$ are continuous and uniformly bounded for each $k$.

Hypothesis 2.II. $\left|f\left\{t, \phi\left(t^{\prime}\right)\right\}\right| \leqq B$ for $0 \leqq t \leqq T$ and $-\infty<t^{\prime}<+\infty$.

HyротнеSIs 2.III. $|f\{t, x\}-f\{t, \bar{x}\}| \leqq A \cdot\|x-\bar{x}\|$, where $(x)$ and $(\bar{x})$ are both points of $R(r)$ and $0 \leqq t \leqq T$.

Then there exists a unique set of functions $x_{1}(t), x_{2}(t), \cdots$, each of class $C^{\prime}$ on the interval $0 \leqq t \leqq K$ ( $K$ is the smaller of the two numbers $T$ and $2^{-1 / p r}$ $/(A r+B))$ with the following two properties:

I. $(x(t))$ belongs to $R$ for $0 \leqq t \leqq K$.

II. If these functions are substituted in (1.1) the right hand members exist in the sense of Lebesgue and are identically equal to the left members for $0 \leqq t \leqq K$.

Such a set of functions will be called a solution.

We first note as a consequence of Hypotheses 2.II and 2.III and (1.2) that

$$
|f\{t, x\}| \leqq A r+B=C \text { for }(x) \text { in } R .
$$

The actual solution is constructed from the following system of successive approximations:

$$
\begin{aligned}
& x_{k}{ }^{(0)}(t)=\phi_{k}(t), \cdots \\
& x_{k}^{(n)}(t)=\phi_{k}(t)+\frac{1}{\mu_{k}} \int_{0}^{t} f_{k}\left\{\tau, x^{(n-1)}(\tau)\right\} \sin \mu_{k}(t-\tau) d \tau \\
& \quad(n=1,2,3, \cdots) .
\end{aligned}
$$

Differentiating these, we have also

$$
\begin{aligned}
& x_{k}{ }^{(0)^{\prime}}(t)=\phi_{k}^{\prime}(t), \cdots, \\
& x_{k}^{(n)^{\prime}}(t)=\phi_{k}^{\prime}(t)+\int_{0}^{t} f_{k}\left\{\tau, x^{(n-1)}(\tau)\right\} \cos \mu_{k}(t-\tau) d \tau .
\end{aligned}
$$

We prove by induction that, for $0 \leqq t \leqq K, x_{k}{ }^{(n)}(t)$ exists and is of class $C^{\prime}$ (cf. Lemma I and its corollary), and that $\left(x^{(n)}(t)\right)$ belongs to $R$. Assuming these facts true for $\left(x^{(n-1)}(t)\right)$, it follows from (2.2), (2.3), and (1.6) that

$$
\begin{aligned}
\left\|x^{(n)}(t)-\phi(t)\right\|^{p}= & \left|\int_{0}^{t} f\left\{\tau, x^{(n-1)}(\tau)\right\} \sin \mu(t-\tau) d \tau\right|^{p} \\
& +\left|\int_{0}^{t} f\left\{\tau, x^{(n-1)}(\tau)\right\} \cos \mu(t-\tau) d \tau\right|^{p}
\end{aligned}
$$




$$
\begin{aligned}
& \leqq 2\left|\int_{0}^{t}\right| f\left\{\tau, x^{(n-1)}(\tau)\right\}|d \tau|^{p} \leqq 2 t^{p-1} \int_{0}^{t}\left|f\left\{\tau, x^{(n-1)}(\tau)\right\}\right|^{p} d \tau \\
& \leqq 2 t^{p-1} \int_{0}^{t} C^{p} d \tau=2 C^{p} t^{p} \leqq r^{p} \text {, i.e. }\left(x^{(n)}(t)\right) \text { belongs to } R .
\end{aligned}
$$

Since the stated facts are obviously true for $x_{k}(0)(t)$, they are by induction true for $x_{k}^{(n)}(t)$.

We next prove that $x_{k}^{(n)}(t)$ converges uniformly toward a limit function $x_{k}(t)$ as $n$ becomes infinite. By setting $n=1$, we have from the above inequalities

$$
\left\|x^{(1)}(t)-x^{(0)}(t)\right\|^{p}=\left\|x^{(1)}(t)-\phi(t)\right\|^{p} \leqq 2 C^{p} t^{p} .
$$

We also obtain from (2.2), (2.3), (1.6), and Hypothesis 2.III

$$
\begin{aligned}
\left\|x^{(n+1)}(t)-x^{(n)}(t)\right\|^{p}= & \left|\int_{0}^{t}\left[f\left\{\tau, x^{(n)}(\tau)\right\}-f\left\{\tau, x^{(n-1)}(\tau)\right\}\right] \sin \mu(t-\tau) d \tau\right|^{p} \\
& +\left|\int_{0}^{t}\left[f\left\{\tau, x^{(n)}(\tau)\right\}-f\left\{\tau, x^{(n-1)}(\tau)\right\}\right] \cos \mu(t-\tau) d \tau\right|^{p} \\
\leqq & 2\left|\int_{0}^{t}\right| f\left\{\tau, x^{(n)}(\tau)\right\}-f\left\{\tau, x^{(n-1)}(\tau)\right\}|d \tau|^{p} \\
\leqq & 2 t^{p-1} \int_{0}^{t}\left|f\left\{\tau, x^{(n)}(\tau)\right\}-f\left\{\tau, x^{(n-1)}(\tau)\right\}\right| p d \tau \\
\leqq & 2 A^{p} t^{p-1} \int_{0}^{t}\left\|x^{(n)}(\tau)-x^{(n-1)}(\tau)\right\| p d \tau .
\end{aligned}
$$

It then follows by induction that

$$
\left\|x^{(n)}(t)-x^{(n-1)}(t)\right\|^{p} \leqq \frac{2^{n} C^{p} A^{p(n-1)} t^{n p}}{(p+1)(2 p+1) \cdots([n-1] p+1)} .
$$

The uniform convergence of $x_{k}^{(n)}(t)$ and $x_{k}^{(n)^{\prime}}(t)$ for $0 \leqq t \leqq K$ now follows from the Weierstrass test.

We also find, using (1.2), that

$$
\begin{aligned}
\left\|x(t)-x^{(n)}(t)\right\| & \leqq \sum_{m=n}^{\infty}\left\|x^{(m+1)}(t)-x^{(m)}(t)\right\| \\
& \leqq \sum_{m=n}^{\infty} \frac{2^{(m+1) / p} C A^{m} K^{m+1}}{[(p+1)(2 p+1) \cdots(m p+1)]^{1 / p}},
\end{aligned}
$$

which is the remainder after $(n-1)$ terms of a certain convergent series of 
positive constants. Now $\left|f\{t, x(t)\}-f\left\{t, x^{(n)}(t)\right\}\right| \leqq A \cdot\left\|x(t)-x^{(n)}(t)\right\|$, so that

$$
\lim _{n=\infty} f_{k}\left\{t, x^{(n)}(t)\right\}=f_{k}\{t, x(t)\}
$$

uniformly for $0 \leqq t \leqq K$.

This is all that is needed to complete the proof that the functions $x_{k}(t)$ satisfy equations (1.1). The proof of the uniqueness of this solution follows essentially the same lines and is left to the reader.

3. Approximation by solutions of finite systems. Let $n$ be a positive integer. Then corresponding to the infinite system of equations (1.1) there is also the finite system,

$$
x_{n k}(t)=\phi_{k}(t)+\frac{1}{\mu_{k}} \int_{0}^{t} \psi_{k}^{(n)}\left\{\tau, x_{n}(\tau)\right\} \sin \mu_{k}(t-\tau) d \tau(k=1,2, \cdots, n)
$$

for determining the unknown functions $x_{n k}(t)$. It is the main object of this article to consider, under certain hypotheses, the approximation to $x_{1}(t)$, $\cdots, x_{n}(t)$, which are the first $n$ functions of the solution of (1.1), by the functions $x_{n 1}(t), x_{n 2}(t), \cdots, x_{n n}(t)$, which form the solution of (3.1). We prove the following

Theorem. Suppose that the limits $|a \mu|$ and $\left|a^{\prime}\right|$ exist, and that there are four positive numbers $r, A, B$, and $T$ independent of $n$ so that the following hypotheses hold:

HyPoTHESIs 3.I. The convergent functions $f_{k}\{t, x\}$ are defined in a region $Q(q)$, where $q=r+2^{1 / p}\left(|a \mu|+\left|a^{\prime}\right|\right)$, and for $0 \leqq t \leqq T$ (see (1.3)). The approximation functions $\psi_{k}^{(n)}$ have the special form $\psi_{k}^{(n)}\{t, x\} \equiv f_{k}\left(t, x_{1}, x_{1}^{\prime}, \cdots\right.$, $\left.x_{n}, x_{n}^{\prime}, 0,0,0, \cdots\right), k=1,2, \cdots$.

HyPoTHESIS 3.II. $\left|\psi^{(n)}\left\{t, \phi\left(t^{\prime}\right)\right\}\right| \leqq B$ for $0 \leqq t \leqq T,-\infty<t^{\prime}<+\infty$.

HyPothesis 3.III. $|f\{t, x\}-f\{t, \bar{x}\}| \leqq A \cdot\|x-\bar{x}\|$ for $0 \leqq t \leqq T$ and for $(x)$ and ( $\bar{x})$ in $Q(q) ; f_{k}\{t, x\}$ is continuous in $t$.

Then we may draw the following four conclusions:

ConCLUSION I. $R(r)<Q(q)$.

CoNCLUSION II. Hypotheses 2.I, 2.II, 2.III of the preceding article hold and consequently a unique solution, $x_{1}(t), x_{2}(t), \cdots$, of (1.1) exists for $0 \leqq t \leqq K$.

ConcLUSION III. A solution $x_{n 1}(t), \cdots, x_{n n}(t)$ of equations (3.1) also exists for $0 \leqq t \leqq K$, such that $\left\|x_{n}(t)-\phi(t)\right\|_{1, n} \leqq r$ and $\left|\psi^{(n)}\left\{t, x_{n}(t)\right\}\right| \leqq A r+B$ $=C$.

CoNCLUSION IV. $\lim _{n=\infty}\left\|x(t)-x_{n}(t)\right\|_{1, n}=0, \lim _{n-\infty}\|x(t)\|_{n+1, \infty}=0, \lim _{n-\infty}$ $\int_{0}^{T}\left|\psi^{(n)}\left\{t, x_{n}(t)\right\}\right|_{n+1, \infty}^{p_{1}} d t=0$, the first two of these limits holding uniformly for $0 \leqq t \leqq K$. 
Conclusions I, II, and III are sufficiently obvious to require no proof. We content ourselves with a proof of IV.

From Hypotheses 3.I and 3.III we have

$$
\begin{aligned}
\left|f\{t, x\}-\psi^{(n)}\{t, \bar{x}\}\right|^{p} \leqq & A^{p}\|x-\bar{x}\|_{1, n}^{p} \\
& +A^{p}\|x\|_{n+1, \infty}^{p} \text { if }(x) \text { and }(\bar{x}) \text { both belong to } Q .
\end{aligned}
$$

Let $\epsilon$ be a preassigned positive number arbitrarily small. Choose $N_{1}$ so large that

$$
|a \mu|_{n+1, \infty}+\left|a^{\prime}\right|_{n+1, \infty} \leqq \epsilon \text { for } n \geqq N_{1} .
$$

Inequality (2.1) holds, and it is easy to justify the relation

$$
\int_{0}^{K}|f\{\tau, x(\tau)\}|^{p} d \tau=\sum_{k=1}^{\infty} \int_{0}^{K}\left|f_{k}\{\tau, x(\tau)\}\right|^{p} d \tau \leqq C^{p} K \quad(C=A r+B) .
$$

Consequently it is possible to find $N_{2}$ so great that

$$
\int_{0}^{K}|f\{\tau, x(\tau)\}|_{n+1, \infty}^{p} d \tau=\sum_{k=n+1}^{\infty} \int_{0}^{K}\left|f_{k}\{\tau, x(\tau)\}\right|^{p} d \tau<\epsilon \text { for } n \geqq N_{\mathbf{2}}
$$

Let $N_{3}$ be the greater of the two numbers $N_{1}$ and $N_{2}$ so that both (3.3) and (3.4) hold as long as $n \geqq N_{3}$. Now, from (1.1) and (1.6), we have

$$
\begin{aligned}
\|x(t)-\phi(t)\|_{n+1, \infty}^{p} & \leqq 2\left|\int_{0}^{t}\right| f\{\tau, x(\tau)\}|d \tau|_{n+1, \infty}^{p} \\
& \leqq 2 K^{p-1} \int_{0}^{K}|f\{\tau, x(\tau)\}|_{n+1, \infty}^{p} d \tau \leqq 2 K^{p-1} \epsilon .
\end{aligned}
$$

Also

$$
\|x(t)\|_{n+1, \infty} \leqq\|x(t)-\phi(t)\|_{n+1, \infty}+\|\phi(t)\|_{n+1, \infty} \leqq\left(2 K^{p-1} \epsilon\right)^{1 / p}+\|\phi(t)\|_{n+1, \infty} .
$$

But since it is easy to show that $\|\phi(t)\|_{n+1, \infty} \leqq 2^{1 / p}\left(|a \mu|_{n+1, \infty}+|a|_{n+1, \infty}\right)$ $\leqq 2^{1 / p_{\epsilon}}$ we have $\|x(t)\|_{n+1, \infty} \leqq\left(2 K^{p-1} \epsilon\right)^{1 / p}+2^{1 / p} \epsilon$ as long as $n \geqq N_{3}$.

Since $N_{3}$ is independent of $t$ for $0 \leqq t \leqq K$, we have proved the second relation under IV.

For convenience choose a number $N_{4}$ so large that

$$
\|x(t)\|_{n+1, \infty}^{p} \leqq \epsilon \text { as long as } n \geqq N_{4} .
$$

Now set up the successive approximations for equations (3.1):

$$
\begin{aligned}
x_{n k}^{(0)}(t) & =\phi_{k}(t), \cdots, \\
x_{n k}^{(m)}(t) & =\phi_{k}(t)+\frac{1}{\mu_{k}} \int_{0}^{t} \psi_{k}^{(n)}\left\{\tau, x_{n}^{(m-1)}(\tau)\right\} \sin \mu_{k}(t-\tau) d \tau, \cdots .
\end{aligned}
$$


We find now from (1.1) and (1.5) that

$$
\left\|x(t)-x_{n}^{(m)}(t)\right\|_{1, n}^{p} \leqq 2 t^{p-1} \int_{0}^{t}\left|f\{\tau, x(\tau)\}-\psi^{(n)}\left\{\tau, x_{n}^{(m-1)}(\tau)\right\}\right|_{1, n}^{p} d \tau,
$$

and this by (3.2) is less than or equal to

$$
2 t^{p-1} \int_{0}^{t} A^{p} \cdot\left\|x(\tau)-x_{n}^{(m-1)}(\tau)\right\|_{1, n}^{p} d \tau+2 t^{p-1} \int_{0}^{t} A^{p} \cdot\|x(\tau)\|_{n+1, \infty}^{p} d \tau .
$$

Therefore we obtain the following inequality:

$$
\text { (3.6) }\left\|x(t)-x_{n}^{(m)}(t)\right\|_{1, n}^{p} \leqq 2 A^{p} t^{p-1} \int_{0}^{t}\left\|x(\tau)-x_{n}{ }^{(m-1)}(\tau)\right\|_{1, n}^{p} d \tau+2 A^{p} t^{p} \epsilon,
$$

which is valid for $0 \leqq t \leqq K$ and $n \geqq N_{4}$. Furthermore it is clear that

$$
\left\|x(\tau)-x_{n}^{(0)}(\tau)\right\|_{1, n}^{p}=\|x(\tau)-\phi(\tau)\|_{1, n}^{p} \leqq r^{p} .
$$

Hence setting $m=1$ in (3.6) we obtain

$$
\left\|x(t)-x_{n}^{(1)}(t)\right\|_{1, n}^{p} \leqq 2 A^{p} r^{p} t^{p}+\epsilon \cdot 2 A^{p} t^{p} .
$$

It is now easy to prove by induction that

$$
\begin{aligned}
&\left\|x(t)-x_{n}^{(m)}(t)\right\|_{1, n}^{p} \leqq \frac{2{ }^{m} A^{m p} r^{p} t^{m p}}{(p+1)(2 p+1) \cdots([m-1] p+1)} \\
&+\epsilon\left[\sum_{j=1}^{m} \frac{2^{j} A^{j p} t^{j p}}{(0 \cdot p+1)(1 \cdot p+1)(2 \cdot p+1) \cdots([j-1] \cdot p+1)}\right] .
\end{aligned}
$$

Since $0 \leqq t \leqq K$, we surely must have now

$$
\left\|x(t)-x_{n}^{(m)}(t)\right\|_{1, n}^{p} \leqq \frac{2^{m} A^{m p} r^{p} K^{m p}}{(p+1)(2 p+1) \cdots([m-1] p+1)}+\epsilon D,
$$

where $D$ is equal to the value of the convergent series of positive constants

$$
\sum_{j=1}^{\infty} \frac{2^{i} A^{j p} K^{i p}}{(0 \cdot p+1)(1 \cdot p+1)(2 \cdot p+1) \cdots([j-1] \cdot p+1)} \cdot
$$

Also it is known that

$$
\lim _{m=\infty} x_{n k}^{(m)}(t)=x_{n k}(t)
$$

uniformly, and the limit of the first term on the right, as $m$ increases indefinitely, is zero. 
Hence $\left\|x(t)-x_{n}(t)\right\|_{1, n} \leqq(\epsilon D)^{1 / p}$, as long as $n \geqq N_{4}$. This proves the first relation under IV.

By (1.2) we have

$$
\begin{aligned}
\int_{0}^{K}\left|\psi^{(n)}\left\{\tau, x_{n}(\tau)\right\}\right|_{n+1, \infty} d \tau & \leqq \int_{0}^{K}|f\{\tau, x(\tau)\}|_{n+1, \infty} d \tau \\
& +\int_{0}^{K}\left|\psi^{(n)}\left\{\tau, x_{n}(\tau)\right\}-f\{\tau, x(\tau)\}\right|_{n+1, \infty} d \tau .
\end{aligned}
$$

We first appraise the first term on the right:

$$
\begin{aligned}
{\left[\int_{0}^{K}|f\{\tau, x(\tau)\}|_{n+1, \infty} d \tau\right]^{p} } & \leqq K^{p-1} \int_{0}^{K}|f\{\tau, x(\tau)\}|_{n+1, \infty}^{p} d \tau \\
& \leqq \epsilon K^{p-1} \text { by }(3.4) \text { for } n \geqq N_{3} .
\end{aligned}
$$

Thus the first term on the right of (3.7) is not greater than $\left(\epsilon K^{p-1}\right)^{1 / p}$ for $n \geqq N_{3}$. The second term on the right of (3.7) is appraised by means of (3.2):

$$
\begin{aligned}
\left|\psi^{(n)}\left\{\tau, x_{n}(\tau)\right\}-f\{\tau, x(\tau)\}\right|_{n, \infty}^{p} & \leqq A^{p} \cdot\left\|x(\tau)-x_{n}(\tau)\right\|_{1, n}^{p}+A^{p} \cdot\|x(\tau)\|_{n+1, \infty}^{p} \\
& \leqq \epsilon A^{p}(D+1) \text { for } n \geqq N_{4} .
\end{aligned}
$$

Thus the second term on the right of (3.7) is not greater than $K A(\epsilon(D+1))^{1 / p}$ for $n \geqq N_{4}$. Therefore

$$
\int_{0}^{K}\left|\psi^{(n)}\left\{\tau, x_{n}(\tau)\right\}\right|_{n+1, \infty} d \tau \leqq \frac{E \epsilon^{1 / p}}{C^{p-1}} \text {, for } n \geqq N_{5},
$$

where $N_{5}$ is the greater of the two numbers $N_{3}$ and $N_{4}$, where $C=A r+B$, and where $E=C^{p-1}\left[K A(D+1)^{1 / p}+K^{(p-1) / p}\right]$. Also, from the obvious relations

$$
\left|\psi^{(n)}\right|_{n+1, \infty}^{p}=\left|\psi^{(n)}\right|_{n+1, \infty}^{p-1} \cdot\left|\psi^{(n)}\right|_{n+1, \infty} \leqq C^{p-1} \cdot\left|\psi^{(n)}\right|_{n+1, \infty}
$$

it follows that

$$
\int_{0}^{K}\left|\psi^{(n)}\left\{\tau, x_{n}(\tau)\right\}\right|_{n+1, \infty}^{p} d \tau \leqq E \epsilon^{1 / p} \text {, as long as } n \geqq N_{5} .
$$

This completes the proof of the theorem.

\section{PART II}

4. The application of the results of Part I to partial differential equations. We consider partial differential equations of the form

$$
P(u) \equiv \frac{\partial^{2} u}{\partial t^{2}}-\frac{\partial^{2} u}{\partial y^{2}}-F\left(\frac{\partial u}{\partial y}, \frac{\partial u}{\partial t}, u, y, t\right)=0,
$$


where $F\left(p_{1}, p_{2}, u, y, t\right)$ is defined for $-\infty<p_{1}<+\infty,-\infty<p_{2}<+\infty,|u| \leqq h$, $0 \leqq y \leqq \pi, 0 \leqq t \leqq T$; is uniformly continuous in $y$ and $t$; and obeys a Lipschitz condition in $p_{1}, p_{2}$, and $u$ :

$$
\begin{aligned}
\left|F\left(p_{1}, p_{2}, u, y, t\right)-F\left(\bar{p}_{1}, \bar{p}_{2}, \bar{u}, y, t\right)\right| \\
\quad \leqq \alpha \cdot\left|p_{1}-\bar{p}_{1}\right|+\beta \cdot\left|p_{2}-\bar{p}_{2}\right|+\gamma \cdot|u-\bar{u}|,
\end{aligned}
$$

as long as $|u| \leqq h,|\bar{u}| \leqq h$.

We retain the notation of $\$ 1$ with the understanding, however, that $p=2$ and $\mu_{k}=k$.

Let

$$
q=\frac{h}{\left|\frac{1}{\mu}\right|}=\frac{h}{\left(\sum_{k=1}^{\infty} \frac{1}{k^{2}}\right)^{1 / 2}}=\frac{6^{1 / 2} h}{\pi} .
$$

Let there be given two functions, $f(y)$ and $g(y)$, defined for the interval $0 \leqq y \leqq \pi$ and subject to the following conditions:

$f(0)=f(\pi)=0 . f(y)$ is an indefinite integral possessing a derivative whose square is summable, and furthermore

$$
\frac{2}{\pi} \int_{0}^{\pi}\left[f^{\prime}(y)\right]^{2} d y<\frac{1}{8} q^{2}
$$

$g(y)$ has a summable square, and furthermore

$$
\frac{2}{\pi} \int_{0}^{\pi}[g(y)]^{2} d y<\frac{1}{8} q^{2} .
$$

We shall try to find a solution of (4.1) such that

$$
u(0, t)=u(\pi, t)=0, \quad u(y, 0)=f(y),\left.\frac{\partial u}{\partial t}\right|_{t=0}=g(y) .
$$

We know from the theory of trigonometric series that

$$
\frac{2}{\pi} \int_{0}^{\pi}\left[f^{\prime}(y)\right]^{2} d y=|a \mu|^{2}<\frac{1}{8} q^{2} \text { where } a_{k} \mu_{k}=a_{k} k=\frac{2}{\pi} \int_{0}^{\pi} f^{\prime}(y) \cos k y d y
$$

and

$$
\frac{2}{\pi} \int_{0}^{\pi}[g(y)]^{2} d y=\left|a^{\prime}\right|^{2}<\frac{1}{8} q^{2} \text { where } a_{k}^{\prime}=\frac{2}{\pi} \int_{0}^{\pi} g(y) \sin k y d y .
$$

Remembering that $f(0)=f(\pi)=0$, we find on integrating by parts that

$$
a_{k}=\frac{2}{\pi} \int_{0}^{\pi} f(y) \sin k y d y .
$$


We also have the obvious inequality $2^{1 / 2}(|a \mu|+|a|)<q$. Let $r=q-2^{1 / 2}(|a \mu|$ $\left.+\left|a^{\prime}\right|\right)$.

Let $(x)$ be a point of $Q(q)$. Then from Schwarz's inequality we have

$$
\sum_{k=1}^{\infty}\left|x_{k}\right| \leqq|x \mu| \cdot\left|\frac{1}{\mu}\right|<\|x\| \cdot\left|\frac{1}{\mu}\right| \leqq q \cdot \frac{\pi}{6^{1 / 2}}=h .
$$

Hence the series $u(y)=\sum_{k=1}^{\infty} x_{k}$ sin $k y$ converges absolutely and uniformly with respect to $y$. With the help of the Riesz-Fischer theorem we may now make the following statement:

Corresponding to a point $(x)$ in $Q$ there is defined a continuous function, $u(y)$, for the interval $0 \leqq y \leqq \pi$, possessing almost everywhere a derivative $u^{\prime}(y)$, whose square is summable, such that

$\mu_{k} x_{k}=\frac{2}{\pi} \int_{0}^{\pi} u^{\prime}(y) \cos k y d y, u(y)=\int_{0}^{y} u^{\prime}(\eta) d \eta, u(0)=u(\pi)=0,|u(y)|<h$.

Corresponding to this same point $(x)$ there is also defined for this interval a second function $v(y)$ whose square is summable and such that

$$
x_{k}^{\prime}=\frac{2}{\pi} \int_{0}^{\pi} v(y) \sin k y d y .
$$

$u^{\prime}(y)$ and $v(y)$ are unique on $0 \leqq y \leqq \pi$ except possibly for point sets of measure zero.

Now let

$$
f_{k}\{t, x\}=\frac{2}{\pi} \int_{0}^{\pi} F\left(u^{\prime}(y), v(y), u(y), y, t\right) \sin k y d y .
$$

Let $[u(y), v(y)]$ and $[\bar{u}(y), \bar{v}(y)]$ be the two pairs of functions corresponding respectively to the two points $(x)$ and $(\bar{x})$ in $Q$. From (4.2) we have

$$
\begin{aligned}
& {\left[F\left(u^{\prime}(y), v(y), u(y), y, t\right)-F\left(\bar{u}^{\prime}(y), \bar{v}(y), \bar{u}(y), y, t\right)\right]^{2}} \\
& \quad \leqq 3 \alpha^{2} \cdot\left[u^{\prime}(y)-\bar{u}^{\prime}(y)\right]^{2}+3 \beta^{2} \cdot[v(y)-\bar{v}(y)]^{2}+3 \gamma^{2} \cdot[u(y)-\bar{u}(y)]^{2} .
\end{aligned}
$$

Now the $f_{k}\{t, x\}$ are the Fourier coefficients of $F\left(u^{\prime}(y), v(y), u(y), y, t\right)$ while the $f_{k}\{t, \bar{x}\}$ are the Fourier coefficients of $F\left(\bar{u}^{\prime}(y), \bar{v}(y), \bar{u}(y), y, t\right)$, and hence the $\left[f_{k}\{t, x\}-f_{k}\{t, \bar{x}\}\right]$ are the Fourier coefficients of

$$
\left[F\left(u^{\prime}(y), v(y), u(y), y, t\right)-F\left(\bar{u}^{\prime}(y), \bar{v}(y), \bar{u}(y), y, t\right)\right] .
$$

Hence 


$$
\begin{aligned}
|f\{t, x\}-f\{t, \bar{x}\}|^{2}= & \frac{2}{\pi} \int_{0}^{\pi}\left[F\left(u^{\prime}(y), v(y), u(y), y, t\right)\right. \\
& \left.-F\left(\bar{u}^{\prime}(y), \bar{v}(y), \bar{u}(y), y, t\right)\right]^{2} d y \\
\leqq & \frac{6 \alpha^{2}}{\pi} \int_{0}^{\pi}\left[u^{\prime}(y)-\bar{u}^{\prime}(y)\right]^{2} d y+\frac{6 \beta^{2}}{\pi} \int_{0}^{\pi}[v(y)-\bar{v}(y)]^{2} d y \\
& +\frac{6 \gamma^{2}}{\pi} \int_{0}^{\pi}[u(y)-\bar{u}(y)]^{2} d y \\
= & 3 \alpha^{2} \cdot|(x-\bar{x}) \mu|^{2}+3 \beta^{2} \cdot\left|x^{\prime}-\bar{x}^{\prime}\right|^{2}+3 \gamma^{2} \cdot|x-\bar{x}|^{2} \\
\leqq & \left(3 \alpha^{2}+3 \gamma^{2}\right) \cdot|(x-\bar{x}) \mu|^{2}+3 \beta^{2} \cdot\left|x^{\prime}-\bar{x}^{\prime}\right|^{2} \\
\leqq & A^{2} \cdot\|x-\bar{x}\|^{2},
\end{aligned}
$$

where $A^{2}$ is the greater of the two numbers $\left(3 \alpha^{2}+3 \gamma^{2}\right)$ and $3 \beta^{2}$. Thus we have

$$
|f\{t, x\}-f\{t, \bar{x}\}| \leqq A \cdot\|x-\bar{x}\| .
$$

Also $|f\{t, x\}| \leqq|f\{t, 0\}|+A \cdot\|x\|$, which is obviously bounded for $(x)$ in $Q$. Finally we write

$$
\psi_{k}^{(n)}\{t, x\} \equiv f_{k}\left(t, x_{1}, x_{1}^{\prime}, \cdots, x_{n}, x_{n}^{\prime}, 0,0,0,0, \cdots\right),
$$

from which it follows, using (4.5), that

$$
\lim _{n=\infty} \psi_{k}^{(n)}\{t, x\}=f_{k}\{t, x\} \text {. }
$$

Thus we easily see that the $f_{k}\{t, x\}$ as here defined satisfy Hypotheses 3.I, 3.II, 3.III. Hence all the results of Part I are now available.

It will be necessary to generalize our idea of a "solution" of a partial differential equation. We first make the following

Definition. A continuous function, $u(y, t)$, defined on the rectangular region $0 \leqq y \leqq \pi, 0 \leqq t \leqq K$, and possessing first partial derivatives almost everywhere in this rectangle, is a solution in the generalized sense of the secondorder partial differential equation $P(u)=0$, if there exists a sequence of functions, $u_{1}(y, t), u_{2}(y, t), u_{3}(y, t), \cdots$, each of class $C^{\prime \prime}$ in this same rectangle, such that the following four conditions hold:

$$
\begin{aligned}
& \lim _{n=\infty} u_{n}(y, t)=u(y, t) \text { uniformly in } y \text { and } t ; \\
& \lim _{n=\infty} \int_{0}^{\pi}\left[\frac{\partial u}{\partial y}-\frac{\partial u_{n}}{\partial y}\right]^{2} d y=0 \text { uniformly in } t ; \\
& \lim _{n=\infty} \int_{0}^{\pi}\left[\frac{\partial u}{\partial t}-\frac{\partial u_{n}}{\partial t}\right]^{2} d y=0 \text { uniformly in } t ;
\end{aligned}
$$




$$
\lim _{n=\infty} \int_{0}^{K} \int_{0}^{\pi}\left[P\left(u_{n}(y, t)\right)\right]^{2} d y d t=0 .
$$

In the next article there will be given a more general definition, which, however, for our present purposes is equivalent to the definition above.

Let $(x(t))$ be a solution, valid for $0 \leqq t \leqq K$, of the infinite system of equations (1.1). We know from $\$ 2$ that such a solution exists and is in fact unique. Then the function

$$
u(y, t)=\sum_{k=1}^{\infty} x_{k}(t) \sin k y
$$

is a solution (in the generalized sense) of (4.1) and satisfies the conditions (4.3). The approximation functions, $u_{n}(y, t)$, mentioned in the definition will be provided for as follows:

$$
u_{n}(y, t)=\sum_{k=1}^{n} x_{n k}(t) \sin k y,
$$

where $x_{n 1}(t), \cdots, x_{n n}(t)$ satisfy the finite system (3.1). Since there is no difficulty about differentiating the finite sum in (4.8), we see that $u_{n}(y, t)$ satisfies the requirement of being of class $C^{\prime \prime}$.

It is intuitively evident that $u(y, t)$ possesses almost everywhere the partial derivatives

$$
\frac{\partial u}{\partial t}=\sum_{k=1}^{\infty} x_{k}^{\prime}(t) \sin k y \text { and } \frac{\partial u}{\partial y}=\sum_{k=1}^{\infty} x_{k}(t) k \cos k y,
$$

where the indicated series converge in the mean on the interval $0 \leqq y \leqq \pi$ for each $t$. They do not necessarily converge in the usual sense. The rigorous proof of these facts is omitted because it merely involves some of the fundamental classical analysis concerning double limits and convergence in the mean.

We notice also that $u(y, t)$ and $\partial u / \partial t$ take on the preassigned initial values of (4.3). For $x_{k}(0)=a_{k}$ and $x_{k}^{\prime}(0)=a_{k}^{\prime}$.

It remains to show that $u(y, t)$ and the $u_{n}(y, t)$ satisfy the Conditions I, II, III, IV of the definition.

Proof that Condition I holds. Let $\epsilon$ be an arbitrarily small positive number. We have

$$
\begin{aligned}
\left|u(y, t)-u_{n}(y, t)\right| & =\left|\sum_{k=1}^{n}\left[x_{k}(t)-x_{n k}(t)\right] \sin k y+\sum_{k=n+1}^{\infty} x_{k}(t) \sin k y\right| \\
& \leqq \sum_{k=1}^{n}\left|x_{k}(t)-x_{n k}(t)\right|+\sum_{k=n+1}^{\infty}\left|x_{k}(t)\right| .
\end{aligned}
$$


Using Schwarz's inequaity we find that

$$
\sum_{k=1}^{n}\left|x_{k}(t)-x_{n k}(t)\right| \leqq\left|\left[x(t)-x_{n k}(t)\right]_{\mu}\right|_{1, n} \cdot\left|\frac{1}{\mu}\right| \leqq\left\|x(t)-x_{n}(t)\right\|_{1, n} \cdot \frac{\pi}{6^{1 / 2}},
$$

and this may be taken less than $\frac{1}{2} \epsilon$, according to the theorem of $\$ 3$, if $n$ is chosen greater than some number $N_{1}$, independent of $t$. Likewise from Schwarz's inequality and the theorem of $\$ 3$ we have

$$
\sum_{k=n+1}^{\infty}\left|x_{k}(t)\right| \leqq|x(t) \mu|_{n+1, \infty} \cdot\left|\frac{1}{\mu}\right| \leqq\|x(t)\|_{n+1, \infty} \cdot \frac{\pi}{6^{1 / 2}} \leqq \frac{1}{2} \epsilon
$$

if $n$ is greater than some number $N_{2}$, independent of $t$. Hence $\mid u(y, t)$ $-u_{n}(y, t) \mid<\epsilon$ for $n>N_{3}$, where $N_{3}$ is the greater of the two numbers $N_{1}$ and $\mathrm{N}_{2}$.

Proof that Condition II holds. The function $\left(\partial u / \partial y-\partial u_{n} / \partial y\right)$ has the Fourier coefficients $\left(x_{k}(t)-x_{n k}(t)\right) k$, for $k=1,2, \cdots, n$, and $x_{k}(t) k$, for $k=n+1, n+2, n+3, \cdots$. Consequently

$$
\begin{aligned}
\frac{2}{\pi} \int_{0}^{\pi}\left(\frac{\partial u}{\partial y}-\frac{\partial u_{n}}{\partial y}\right)^{2} d y & =\left|\left[x(t)-x_{n}(t)\right] \mu\right|_{1, n}^{2}+|x(t) \mu|_{n+1, \infty}^{2} \\
& \leqq\left\|x(t)-x_{n}(t)\right\|_{1, n}^{2}+\|x(t)\|_{n+1, \infty}^{2}
\end{aligned}
$$

and this by $\$ 3$ converges uniformly to zero.

Proof that Condition III holds. The function $\left(\partial u / \partial t-\partial u_{n} / \partial t\right)$ has the Fourier coefficients $x_{k}^{\prime}(t)-x_{n k}^{\prime}(t)$, for $k=1, \cdots, n$, and $x_{k}^{\prime}(t)$, for $k=n+1$, $n+2, \cdots$. Consequently

$$
\begin{aligned}
\frac{2}{\pi} \int_{0}^{\pi}\left(\frac{\partial u}{\partial t}-\frac{\partial u_{n}}{\partial t}\right)^{2} d y & =\left|x^{\prime}(t)-x_{n}^{\prime}(t)\right|_{1, n}^{2}+\left|x^{\prime}(t)\right|_{n+1, \infty}^{2} \\
& \leqq\left\|x(t)-x_{n}(t)\right\|_{1, n}^{2}+\|x(t)\|_{n+1, \infty}^{2}
\end{aligned}
$$

which converges uniformly to zero as above.

Proof that Condition IV holds. Differentiate $u_{n}(y, t)$ and substitute in the operator, $P(\quad) . P\left[u_{n}(y, t)\right]$ thus is a function of $y$ and $t$. It is found from (4.1), (4.4), and (4.6) that

$$
\frac{2}{\pi} \int_{0}^{\pi} P\left[u_{n}(y, t)\right] \sin k y d y=x_{n k}^{\prime \prime}(t)+k^{2} x_{n k}-\psi_{k}^{(n)}\left\{t, x_{n}(t)\right\},
$$

$$
\text { for } k=1,2, \cdots, n \text {, }
$$

and this vanishes on account of (3.1). Also

$$
\begin{array}{r}
\frac{2}{\pi} \int_{0}^{\pi} P\left[u_{n}(y, t)\right] \sin k y d y=-\psi_{k}^{(n)}\left\{t, x_{n}(t)\right\} \\
\text { for } k=n+1, n+2, n+3, \cdots .
\end{array}
$$


Hence, by Parseval's theorem,

$$
\frac{2}{\pi} \int_{0}^{\pi}\left\{P\left[u_{n}(y, t)\right]\right\}^{2} d y=\left|\psi^{(n)}\left\{t, x_{n}(t)\right\}\right|_{n+1, \infty}^{2}
$$

and integrating this with respect to $t$ between the limits 0 and $K$ we get a quantity which, by the theorem of $\S 3$, may be taken arbitrarily small by taking $n$ sufficiently large.

5. A study of the conception of a "solution in the generalized sense" with special reference to partial differential equations of the form (4.1). It will be shown in this section that a solution, $u(y, t)$, of $(4.1)$ in the generalized sense is also a solution in the ordinary sense, provided thac $u$ possesses second derivatives and $F$ satisfies certain simple requirements as to continuity and differentiability. It will also be shown that the solution in the generalized sense obtained in the previous section is the only such solution which satisfies the boundary conditions (4.3).

It is easy to prove these theorems for equation (4.1) because of its especially simple structure. But the generalized notion of a solution of a partial differential equation is naturally of a much broader character. It might very well prove useful in the treatment of all partial differential equations, especially those of the hyperbolic type. I give here a complete definition, slightly more general than the one introduced in $\$ 4$, which was not symmetrical in $y$ and $t$.

Definition 1. A continuous function, $u(y, t)$, defined in some finite region $E$ and possessing almost everywhere in $E$ first partial derivatives, is a solution in the generalized sense of the second-order partial differential equation $P(u)=0$ if there exists a sequence of functions, $u_{1}(y, t), u_{2}(y, t), u_{3}(y, t), \cdots$, each defined in $E$ and each of class $C^{\prime \prime}$, such that the following four conditions hold:

$$
\begin{aligned}
& \lim _{n=\infty} u_{n}(y, t)=u(y, t) \text { uniformly in } E ; \\
& \lim _{n=\infty} \iint_{E}\left|\frac{\partial u}{\partial y}-\frac{\partial u_{n}}{\partial y}\right| d y d t=0 ; \\
& \lim _{n=\infty} \iint_{E}\left|\frac{\partial u}{\partial t}-\frac{\partial u_{n}}{\partial t}\right| d y d t=0 ; \\
& \lim _{n=\infty} \iint_{E}\left|P\left(u_{n}\right)\right| d y d t=0 .
\end{aligned}
$$

In the sequel, $E$ will be assumed to be a closed simply connected region, whose boundary consists (say) of a finite number of arcs of analytic curves. 
In comparing this definition with the one given in $\$ 4$, it will be observed that the exponent 2 has been omitted from the integrands in II, III, IV. However, Schwarz's inequality shows us that, if

then also

$$
\lim _{n=\infty} \iint_{E}\left|\Psi_{n}\right|^{2} d y d t=0,
$$

$$
\lim _{n=\infty} \iint_{E}\left|\Psi_{n}\right| d y d t=0,
$$

provided that $E$ is finite. In other words, a function $u(y, t)$ which satisfies the conditions of the former definition will surely satisfy the conditions of this last definition.

From the fundamental facts about convergence on the average, the reader will readily verify the truth of the following

Lemma 1. Let $\left(y^{\prime}, t^{\prime}\right)$ be an interior point of $E$ and $\delta$ a sufficiently small preassigned positive number. Then it is possible to find a subsequence, $u_{n}^{*}(y, t)$, of the sequence $u_{n}(y, t)$, such that for almost all choices of $t_{0}$ in the interval $\left|t^{\prime}-t_{0}\right| \leqq \delta$ we shall have

$$
\lim _{k=\infty} \int_{E_{t_{0}}}\left|\frac{\partial u}{\partial t}-\frac{\partial u_{k}^{*}}{\partial t}\right| d y=0,
$$

where $E_{t_{0}}$ denotes the cross-sectional point set obtained from $E$ by putting $t=t_{0}$, and where $u(y, t)$ and $u_{n}(y, t)$ satisfy the requirements of Definition 1 .

Definition 2. Let $u(y, t)$, defined on $E$, be a solution of $P(u)=0$ in the generalized sense, and let $u_{n}(y, t)$ be the approximation functions introduced in Definition 1 . Then the cross-sectional point set $E_{t_{0}}$ obtained from $E$ by setting $t=t_{0}$ is called a proper line, if there exists a subsequence $u_{n}^{*}(y, t)$ such that

$$
\lim _{n=\infty} \int_{E_{t_{0}}}\left|\frac{\partial u}{\partial t}-\frac{\partial u_{n}^{*}}{\partial t}\right| d y=0 .
$$

If such a subsequence does not exist, $E_{t_{0}}$ is called an improper line.

According to Lemma 1, almost all cross-sections are proper lines.

Definition 3. A solution, $u(y, t)$, of $P(u)=0$ in the generalized sense will be said to assume the initial values $f(y)$ and $g(y)$ for $t=t_{0}$, if both the following conditions hold:

(I) $E_{t_{0}}$ is a proper line;

(II) $u\left(y, t_{0}\right)=f(y)$, and

almost everywhere on $E_{t_{0}}$.

$$
\left.\frac{\partial u}{\partial t}\right|_{t=t_{0}}=g(y)
$$


The subsequence $u_{n}^{*}(y, t)$ mentioned in Definition 2 will then be such that

$$
\lim _{n=\infty} \int_{E_{t_{0}}}\left|\frac{\partial u_{n}^{*}}{\partial t}-g(y)\right| d y=0 .
$$

We temporarily consider the simple equation

$$
\frac{\partial^{2} u}{\partial t^{2}}-\frac{\partial^{2} u}{\partial y^{2}}=\phi(y, t)
$$

LEMMA 2. If $u(y, t)$ is a given function satisfying (5.1) in the ordinary sense, then

$$
\begin{aligned}
u(y, t)= & \frac{1}{2} u\left(y+t-t_{0}, t_{0}\right)+\frac{1}{2} u\left(y-t+t_{0}, t_{0}\right) \\
& +\frac{1}{2} \int_{y-t+t_{0}}^{y+t-t_{0}} \frac{\partial u}{\partial t}\left(\eta, t_{0}\right) d \eta+\frac{1}{2} \iint_{\operatorname{tri}\left(y, t, t_{0}\right)} \phi(\eta, \tau) d \eta d \tau .
\end{aligned}
$$

$t_{0}$ is arbitrary except as restricted below. The region of integration for the double integral on the right, denoted by tri $\left(y, t, t_{0}\right)$; is the triangle in the $(\eta, \tau)$ plane with vertices at the following points: $(y, t),\left(y-t+t_{0}, t_{0}\right)$, and $\left(y+t-t_{0}, t_{0}\right)$.

We also require that $\operatorname{tri}\left(y, t, t_{0}\right)$ shall lie entirely within the region of definition of $\phi$ and $u$, and that $\phi$ shall be integrable, so that the right hand side of (5.2) will have a meaning.

This well known lemma can be easily proved by applying the following formula, deduced from Green's theorem, to tri $\left(y, t, t_{0}\right)$ :

$$
\iint_{S}\left(\frac{\partial^{2} u}{\partial y^{2}}-\frac{\partial^{2} u}{\partial t^{2}}\right) d \eta d \tau=\int_{C} \frac{\partial u}{\partial t} d \eta+\frac{\partial u}{\partial y} d \tau
$$

where $S$ represents any closed region in the $(\eta, \tau)$ plane and $C$ represents the boundary of $S$ taken in the proper sense. For later convenience I have written $\eta$ and $\tau$ as the variables of integration instead of $y$ and $t$. That is, in the above integrals, I regard $u(y, t)$ and its partial derivatives as being evaluated for $y=\eta$ and $t=\tau$. In the sequel, the notation will frequently be changed in this way, whenever no confusion is likely to result, with no further comment. The actual proof of the lemma is omitted.

From Lemma 2 we see that the non-linear partial differential equation (4.1) is closely related to the following non-linear integro-partial differential equation:

$$
\begin{gathered}
u(y, t)=\frac{1}{2} u\left(y+t-t_{0}, t_{0}\right)+\frac{1}{2} u\left(y-t+t_{0}, t_{0}\right) \\
+\left.\frac{1}{2} \int_{\nu-t+t_{0}}^{y+t-t_{0}} \frac{\partial u}{\partial t}\right|_{\tau=t_{0}} d \eta+\frac{1}{2} \iint_{\operatorname{tri}\left(y, t, t_{0}\right)} F\left[\frac{\partial u}{\partial y}, \frac{\partial u}{\partial t}, u(\eta, \tau), \eta, \tau\right] d \eta d \tau
\end{gathered}
$$

which we now proceed to consider. 
Definition 4. A function $u(y, t)$, defined in $E$ and admitting almost everywhere jirst partial derivatives, is a solution of (5.3) at the point $\left(y^{\prime}, t^{\prime}\right)$ in $E$, if, for almost all values of $t_{0}$ (termed "proper" values) in the neighborhood of $t^{\prime}$, (5.3) is an identity in $y$ and $t$ in the neighborhood of $\left(y^{\prime}, t^{\prime}\right)$. It is a solution throughout $E$, if it is a solution at every interior point of $E$.

Equations (4.1) and (5.3) are only partially equivalent because a solution of (4.1) in the ordinary sense must possess second derivatives, whereas a solution of (5.3) need not possess second derivatives. We shall see, however, that (4.1) and (5.3) are completely equivalent, if by a solution of (4.1) we mean a solution in the generalized sense, provided that $F$ shall satisfy certain simple conditions.

THEOREM 1. Let $F\left(p_{1}, p_{2}, u, y, t\right)$ be defined for all $p_{1}$ and $p_{2}$, for $|u| \leqq h$, and for $(y, t)$ in $E$. Let $F$ also obey the Lipschitz condition

$$
|F(p, p, u, y, t)-F(\tilde{p}, \tilde{p}, \tilde{u}, y, t)| \leqq \alpha\left|p_{1}-\tilde{p}_{1}\right|+\beta\left|p_{2}-\tilde{p}_{2}\right|+\gamma|u-\tilde{u}|
$$

for $|u| \leqq h$ and $|u| \leqq h$. Then a solution $u(y, t)$ of (4.1) in the generalized sense, such that $|u(y, t)|<h$, is also a solution of (5.3).

There exists a sequence of functions $u_{n}(y, t)$ satisfying the conditions of Definition 1. Because of Condition I we may assume without loss of generality that $\left|u_{n}\right|<h, n=1,2,3, \cdots$.

Let $\left(y^{\prime}, t^{\prime}\right)$ be any interior point of $E$. It is possible to choose a positive number $\delta$ and a neighborhood $U$ of $\left(y^{\prime}, t^{\prime}\right)$ such that, if $(y, t)$ is a point of $U$ and $t_{0}$ satisfies the inequality $\left|t^{\prime}-t_{0}\right| \leqq \delta$, the triangular region $\operatorname{tri}\left(y, t, t_{0}\right)$ will lie completely imbedded in $E$.

In accordance with Lemma 1 we have for almost all choices of $t_{0}$ the following relation:

$$
\lim _{n=\infty} \int_{E_{t_{0}}}\left|\frac{\partial u}{\partial t}-\frac{\partial u_{n}^{*}}{\partial t}\right| d y=0,
$$

where $u_{n}^{*}(y, t)$ is a certain subsequence of the given sequence, $u_{n}(y, t)$. In other words, $t=t_{0}$ is a proper line. Choose any such proper line such that $\left|t^{\prime}-t_{0}\right| \leqq \delta$. Then hold $t_{0}$ fast.

If we set

$$
\zeta_{n}(y, t) \equiv \frac{\partial^{2} u_{n}^{*}}{\partial t^{2}}-\frac{\partial^{2} u_{n}^{*}}{\partial y^{2}}-F\left(\frac{\partial u_{n}^{*}}{\partial y}, \frac{\partial u_{n}^{*}}{\partial t}, u_{n}^{*}, y, t\right),
$$

we know from Definition 1, Condition IV, that 


$$
\lim _{n=\infty} \iint_{E}\left|\zeta_{n}(\eta, \tau)\right| d \eta d \tau=0
$$

Also from Lemma 2 we have the following identity in $y$ and $t$ in the neighborhood $U$ of $\left(y^{\prime}, t^{\prime}\right)$ :

$$
\begin{aligned}
u_{n}^{*}(y, t) \equiv & \frac{1}{2} u_{n}^{*}\left(y+t-t_{0}, t_{0}\right)+\frac{1}{2} u_{n}^{*}\left(y-t+t_{0}, t_{0}\right) \\
& +\frac{1}{2} \int_{y-t+t_{0}}^{y+t-t_{0}} \frac{\partial u_{n}^{*}}{\partial t}\left(\eta, t_{0}\right) d \eta \\
& +\frac{1}{2} \iint_{\operatorname{tri}\left(y, t, t_{0}\right)} F\left[\frac{\partial u_{n}^{*}}{\partial y}, \frac{\partial u_{n}^{*}}{\partial t}, u^{*}, \eta, \tau\right] d \eta d \tau \\
& +\frac{1}{2} \iint_{\operatorname{tri}\left(y, t, t_{0}\right)} \zeta_{n}(\eta, \tau) d \eta d \tau .
\end{aligned}
$$

Now consider the function

$$
\begin{aligned}
& w(y, t) \equiv \frac{1}{2} u\left(y+t-t_{0}, t_{0}\right)+\frac{1}{2} u\left(y-t+t_{0}, t_{0}\right) \\
& +\frac{1}{2} \int_{y-t+t_{0}}^{y+t-t_{0}} \frac{\partial u}{\partial t}\left(\eta, t_{0}\right) d \eta+\frac{1}{2} \iint_{\operatorname{tri}\left(y, t, t_{0}\right)} F\left[\frac{\partial u}{\partial y}, \frac{\partial u}{\partial t}, u, \eta, \tau\right] d \eta d \tau .
\end{aligned}
$$

The fact that the double integral on the right actually exists follows from the fact that

$$
\iint_{E} F\left[\frac{\partial u_{n}}{\partial y}, \frac{\partial u_{n}}{\partial t}, u_{n}, \eta, \tau\right] d \eta d \tau
$$

is assumed to exist, since $u$ was by hypothesis a solution in the generalized sense. It is easily proved in virtue of the Lipschitz condition and Conditions I, II, and III of Definition 1 that $F\left(\partial u_{n} / \partial y, \partial u_{n} / \partial t, u_{n}(y, t), y, t\right)$ converges in the mean, as $n$ tends to infinity, to $F(\partial u / \partial y, \partial u / \partial t, u(y, t), y, t)$, and hence this latter function is integrable.

We shall show that $w(y, t) \equiv u(y, t)$ in the neighborhood $U$ of $\left(y^{\prime}, t^{\prime}\right)$. Subtracting (5.6) from (5.7) we get

$$
\begin{aligned}
w(y, t)-u_{n}^{*}(y, t)=\frac{1}{2}\{ & \left.u\left(y+t-t_{0}, t_{0}\right)-u_{n}^{*}\left(y+t-t_{0}, t_{0}\right)\right\}+\frac{1}{2}\left\{u\left(y-t+t_{0}, t_{0}\right)\right. \\
& \left.-u_{n}^{*}\left(y-t+t_{0}, t_{0}\right)\right\} \\
& +\frac{1}{2} \int_{y-t+t_{0}}^{v+t-t_{0}}\left\{\frac{\partial u}{\partial t}\left(\eta, t_{0}\right)-\frac{\partial u_{n}^{*}}{\partial t}\left(\eta, t_{0}\right)\right\} d \eta \\
& +\frac{1}{2} \iint_{t r i\left(y, t, t_{0}\right)}\left\{F\left(\frac{\partial u}{\partial y}, \ldots\right)-F\left(\frac{\partial u_{n}^{*}}{\partial y}, \ldots\right)\right\} d \eta d \tau \\
& -\frac{1}{2} \iint_{\operatorname{tri}\left(y, t, t_{0}\right)} \zeta_{n}(\eta, \tau) d \eta d \tau .
\end{aligned}
$$


Let $\epsilon$ be an arbitrarily small positive number. Then the sum of the absolute values of the first two terms on the right can be taken less than $\frac{1}{4} \epsilon$ by taking $n$ sufficiently large (independently of $y$ or $t$ ) because of Condition I of Definition 1 . The absolute value of the third term can be taken less than $\frac{1}{2} \epsilon$ because of (5.4). The absolute value of the fifth (last) term can also be taken less than $1 \epsilon$ by (5.5). And finally applying the Lipschitz condition to the fourth term we have

$$
\begin{aligned}
\left|w(y, t)-u_{n}^{*}(y, t)\right|<\frac{3}{4} \epsilon & +\frac{1}{2} \alpha \cdot \iint_{\operatorname{tri}\left(y, t, t_{0}\right)}\left|\frac{\partial u}{\partial y}-\frac{\partial u_{n}^{*}}{\partial y}\right| d \eta d \tau \\
& +\frac{1}{2} \beta \cdot \iint_{\operatorname{tri}\left(y, t, t_{0}\right)}\left|\frac{\partial u}{\partial t}-\frac{\partial u_{n}^{*}}{\partial t}\right| d \eta d \tau \\
& \left.+\frac{1}{2} \gamma \cdot \iint_{\operatorname{tri}\left(y, t, t_{0}\right)} \mid u(\eta, \tau)-u_{n}^{*} \eta, \tau\right) \mid d \eta d \tau .
\end{aligned}
$$

Hence from Conditions I, II, III, we have $\left|w(y, t)-u_{n}^{*}(y, t)\right|<\epsilon$, if $n>N^{\prime}$ where $N$ is a number depending only upon $\epsilon$. In other words $u_{n}^{*}(y, t)$ tends uniformly to $w(y, t)$ in $U$. Since, however, $u_{n}{ }^{*}$ is a subsequence of $u_{n}$, which by hypothesis converges uniformly to $u(y, t)$, it follows that $w(y, t) \equiv u(y, t)$ in the neighborhood of $\left(y^{\prime}, t^{\prime}\right)$.

Hence $u$ is a solution of (5.3) at the point $\left(y^{\prime}, t^{\prime}\right)$. Since $\left(y^{\prime}, t^{\prime}\right)$ was any interior point of $E, u$ is by Definition 4 a solution throughout $E$ of (5.3).

THEOREM 2. Let $y^{\prime}, t^{\prime}, t_{1}$ be any three real numbers determining a closed triangular region, $\operatorname{tri}\left(y^{\prime}, t^{\prime}, t_{1}\right)$, and such that $t^{\prime}-t_{1}=T>0$. Let $F\left(p_{1}, p_{2}, u, y, t\right)$ be defined for all values of $y$ and $t$ which are coordinates of points in tri $\left(y^{\prime}, t^{\prime}, t_{1}\right)$; for $|u|<h$, and for all values of $p_{1}$ and $p_{2}$ whatever. And suppose that it satisfies the Lipschitz condition

$$
\begin{aligned}
& \left|F\left(\tilde{p}_{1}, \tilde{p}_{2}, \tilde{u}, y, t\right)-F\left(p_{1}, p_{2}, u, y, t\right)\right| \leqq \alpha\left|\tilde{p}_{1}-p_{1}\right|+\beta\left|\tilde{p}_{2}-p_{2}\right|+\gamma|\tilde{u}-u|, \\
& \text { for }|u|<h,|\tilde{u}|<h .
\end{aligned}
$$

Let $f(y)$ and $g(y)$ be defined for $y^{\prime}-T \leqq y \leqq y^{\prime}+T$. Let $f(y)$ be an indefinite integral of a function $f^{\prime}(y)$, and let $g(y)$ be summable.

Then there can not be more than one solution, $u(y, t)$, defined on $\operatorname{tri}\left(y^{\prime}, t^{\prime}, t_{1}\right)$, of the equation

$$
\begin{aligned}
u(y, t) & =\frac{1}{2} f\left(y+t-t_{1}\right)+\frac{1}{2} f\left(y-t+t_{1}\right) \\
& +\frac{1}{2} \int_{y-t+t_{1}}^{v+t-t_{1}} g(\eta) d \eta+\frac{1}{2} \iint_{\operatorname{tri}\left(y, t, t_{1}\right)} F\left[\frac{\partial u}{\partial y}, \frac{\partial u}{\partial t}, u, \eta, \tau\right] d \eta d \tau,
\end{aligned}
$$

for which $|u|<h$. 
Let $u(y, t)$ and $v(y, t)$ be two such solutions of (5.8).

Differentiating, using the Lipschitz condition, and executing other obvious operations, we establish

$$
\begin{aligned}
& 2 \cdot|u(y, t)-v(y, t)| \\
& \left.\int_{y^{\prime}-t^{\prime}+t}^{y^{\prime}+t^{\prime}-t}\left|\frac{\partial u}{\partial t}(\eta, t)-\frac{\partial v}{\partial t}\right| d \eta\right\} \leqq \int_{t_{1}}^{t} \int_{y^{\prime}-t^{\prime}+\tau}^{v^{\prime}+t^{\prime}-\tau}\left\{\alpha \cdot\left|\frac{\partial u}{\partial y}-\frac{\partial v}{\partial y}\right|+\beta \cdot\left|\frac{\partial u}{\partial t}-\frac{\partial v}{\partial t}\right|\right. \\
& \left.\int_{y^{\prime}-t^{\prime}+t}^{y^{\prime}+t^{\prime}-t}\left|\frac{\partial u}{\partial y}(\eta, t)-\frac{\partial v}{\partial y}\right| d \eta|+\gamma \cdot| u-v \mid\right\} d \eta d \tau \text {. }
\end{aligned}
$$

The last two of the above three inequalities hold for almost all values of $t$ on $t_{1} \leqq t \leqq t^{\prime}$. Let $t_{2}$ be one of these non-exceptional values, not greater than $t_{1}+\frac{1}{2} /(\alpha+\beta+T \gamma)$. Let $M$ be the least common upper bound of the left members of the above inequalities for the non-exceptional values of $t$ on the interval $t_{1} \leqq t \leqq t_{2}$ and for $y$ restricted so that the point $(y, t)$ lies in $\operatorname{tri}\left(y^{\prime}, t^{\prime}, t_{1}\right)$. Hence, for some such point $\left(y^{*}, t^{*}\right)$, one at least of these left members is not less than $\frac{3}{4} M$, and we thus obtain

$$
\begin{aligned}
\frac{3}{4} M & \leqq M(\alpha+\beta)\left(t_{2}-t_{1}\right)+2 T\left(t_{2}-t_{1}\right) \gamma M / 2 \\
& =\left(t_{2}-t_{1}\right)(\alpha+\beta+T \gamma) M \leqq \frac{1}{2} M .
\end{aligned}
$$

Therefore $M=0$.

It follows that $u(y, t) \equiv v(y, t)$ in the part of $\operatorname{tri}\left(y^{\prime}, t^{\prime}, t_{1}\right)$ which lies below the line $t=t_{2}$. By a repetition of this argument the reader can readily extend this result to include the whole of tri $\left(y^{\prime}, t^{\prime}, t_{1}\right)$.

Evidently Theorems 1 and 2 can be used to prove the uniqueness of the function $u(y, t)$ of $\$ 4$, which satisfies (in the generalized sense) the differential equation (4.1) and obeys the conditions (4.3).

In order to apply Theorem 2, however, it is first necessary to extend the definitions of $F\left(p_{1}, p_{2}, u, y, t\right), f(y), g(y)$, and $u(y, t)$, which in $\$ 4$ were regarded as defined only for $0 \leqq y \leqq \pi$. It is necessary to do this so that every point in the rectangle $0 \leqq y \leqq \pi, 0 \leqq t \leqq K$ may be imbedded within a triangular region $\operatorname{tri}\left(y^{\prime}, t^{\prime}, 0\right)$. This extension can obviously be effected in a variety of ways. For example, we could define $F, f, g, u$ outside of $0 \leqq y \leqq \pi$ by making them periodic in $y$ with period $\pi$. This definition alone may give conflicting values for $F$ at points for which $y=k \pi, k=0, \pm 1, \pm 2, \pm 3, \cdots$. So we shall redefine $F$ at these points by writing $F\left(p_{1}, p_{2}, u, k \pi, t\right) \equiv 0$. With this extended definition, $u(y, t)$ furnishes us with a generalized solution of (4.1) which is valid for $-N \pi \leqq y \leqq+N \pi, 0 \leqq t \leqq K$, where $N$ is an arbitrarily large integer. In order to see this it is only necessary first to extend the definitions of the approximation functions $u_{n}(y, t)$ by making them periodic in 
$y$ and secondly to modify them slightly near the points for which $y=k \pi$, so that they may be of class $C^{\prime \prime}$ throughout in accordance with Definition 1.

\section{PART III}

6. Statement of the problem for the parabolic partial differential equation. We now treat certain partial differential equations of parabolic type by the methods developed in Parts I and II. A different point of view is assumed, however, in that no use is made of a solution in a generalized sense. M. R. Siddiqi†, using the methods of Lichtenstein, has treated parabolic equations of a more restricted type and for less general initial conditions. The present methods are also simpler than Siddiqi's; but on the other hand Siddiqi's solution is valid for $0 \leqq t<\infty$, whereas the present solution is defined only for a sufficiently small interval $0 \leqq t \leqq K>0$. The inequalities upon which the present work is based seem to admit considerable latitude, but $I$ have been unable to obtain the extension to the infinite interval.

Since practically no repetition is involved, this part of the paper is written so that it can be read independently of Parts I and II.

We shall consider a partial differential equation of the form,

$$
\frac{\partial u}{\partial t}=\frac{\partial^{2} u}{\partial y^{2}}+F\left(\frac{\partial u}{\partial y}, u, y, t\right)
$$

where $F(p, u, y, t)$ is defined for $|p| \leqq P,|u| \leqq U, 0 \leqq y \leqq \pi, 0 \leqq t \leqq T$. It is continuous and possesses continuous partial derivatives with respect to $p, u$, and $y$. Furthermore we assume either that

$$
F(p, 0, y, t) \equiv 0
$$

or that

$$
F(p, u, 0, t) \equiv F(p, u, \pi, t) \equiv 0 . \ddagger
$$

As a consequence of the existence of the continuous partial derivatives we may also write the following Lipschitz condition:

$$
|F(p, u, y, t)-F(\bar{p}, \bar{u}, y, t)| \leqq \alpha \cdot|p-\bar{p}|+\beta \cdot|u-\bar{u}|,
$$

which is valid for the domain of definition specified above.

We have given a function $f(y)$, defined on $0 \leqq y \leqq \pi$, vanishing at the end

$\dagger$ See bibliography given in the Introduction.

$\ddagger$ In the equations treated by Siddiqi, $F$ is developable in a power series in $u$ and $p$ :

$$
F(p, u, y, t)=\sum_{\alpha, \beta} g_{\alpha \beta}(y, t) u^{\alpha} p^{\beta},
$$

where, however, $\alpha$ is not allowed to take on the value 0 . Consequently Siddiqi's equations satisfy (6.2). 
points of this interval, possessing an absolutely continuous first derivative, and having almost everywhere on $0 \leqq y \leqq \pi$ a second derivative whose square is summable. This is equivalent to the assumption that $f(y)$ can be developed in a trigonometric series $f(y)=\sum_{k=1}^{\infty} a_{k} \sin k y$, for which $\sum a_{k}^{2} k^{4}$ converges. $\dagger$ We wish to find a function $u(y, t)$, defined for $0 \leqq y \leqq \pi, 0 \leqq t \leqq K$, which satisfies (6.1) and the boundary conditions

$$
u(y, 0) \equiv f(y), u(0, t) \equiv u(\pi, t) \equiv 0 \quad(0<K \leqq T) .
$$

7. The related infinite systems of ordinary differential equations. We shall here prove an existence lemma for infinite systems of differential equations of the type

$$
\frac{d x_{k}}{d t}+\mu_{k}^{2} x_{k}=f_{k}\{t, x\} \quad(k=1,2,3, \cdots)
$$

under the initial conditions $x_{k}(0)=a_{k}$. Here, contrary to the notation of $\$ 1$, a letter followed by $\{t, x\}$ denotes a function dependent upon the infinitely many independent variables $t, x_{1}, x_{2}, x_{3}, \cdots$. The $\mu_{k}$ are any infinite set of real numbers, such that $1 \leqq \mu_{k} \leqq \mu_{k+1}$. We shall consider (7.1) in the form of the equivalent infinite system of integral equations:

$$
x_{k}(t)-a_{k} e^{-\mu_{k}^{2} t}=\int_{0}^{t} f_{k}\{\tau, x(\tau)\} e^{\mu_{k}^{2}(\tau-t)} d \tau .
$$

As in Part I, we shall use the following terminology and abbreviations:

$[x]$ stands for the infinite system of numbers represented by the symbols $x_{1}, x_{2}, x_{3}, \cdots|x|$ is an abbreviation for $\left(\sum_{k=1}^{\infty} x_{k}^{2}\right)^{1 / 2}$, if this limit exists. It will be noted that this symbol obeys the inequality

$$
\left|x+x^{\prime}\right| \leqq|x|+\left|x^{\prime}\right| \text {. }
$$

The ordered sequence $[x]$ will frequently be regarded as a point in function space. The region $Q(q)$ will consist of those points $[x]$ for which

$$
\left|\mu^{2} x\right| \leqq q,
$$

where $q$ is positive. The region $R(r)$ consists of those points $[x]$ for which

$$
\left|\mu^{2}\left(x-a e^{-\mu^{2} t}\right)\right| \leqq r
$$

for at least one value of $t>0(r>0)$.

We shall always assume that $\left|\mu^{2} a\right|$ exists and we shall take $q=r+\left|\mu^{2} a\right|$. Evidently, then, $R<Q$, as follows from (7.3). $\dagger$ Siddiqi assumes for one of his theorems that $\sum k^{2}\left|a_{k}\right|$ converges and for the other theorem that
$\sum k^{3}\left|a_{k}\right|$ converges. 
We assume that $f_{k}\{t, x\}$ is defined for $0 \leqq t \leqq T$ and for $[x]$ in some region $Q(q)$. Furthermore we assume the following three fundamental hypotheses:

HypothesIS 1. If $[x(t)]$ is any sequence of continuous functions whose range is in $Q(q)$ for $0 \leqq t \leqq T$, then $f_{k}\{t, x(t)\}$ is integrable on $0 \leqq t \leqq T$.

HyротнеSis 2. A number $B$ exists such that $|\mu f\{t, x\}| \leqq 2^{1 / 2} B$ for $[x]$ in $Q(q)$ and for $0 \leqq t \leqq T$.

Hypothesis 3. A number $A$ exists such that $\left|f\{t, x\}-f\{t, \bar{x}\} \leqq 2^{1 / 2} A \cdot\right| \mu(x$ $-\bar{x}) \mid$ for $0 \leqq t \leqq T$ and for $[x]$ and $[\bar{x}]$ in $Q(q)$.

On the basis of these hypotheses we shall prove the existence of an infinite sequence of continuous functions $[x(t)]$ defined for $0 \leqq t \leqq K$ ( $K$ is the smaller of the two numbers $T$ and $\left.r^{2} / B^{2}\right)$ whose range is in $R(r)$, such that when these functions are substituted in (7.2) the right hand members exist and are identically equal to the left. Such a set of functions is called a solution of (7.2). This solution is unique.

From Schwarz's inequality and Lebesgue's theorem for integrating infinite sequences, it is readily verified that, if $F_{k}(t)$ is a sequence of integrable functions such that $\sum_{k=1}^{n} \mu_{k}^{2}\left[F_{k}(t)\right]^{2}$ is uniformly bounded with respect to $n$ and $t$ (for $0 \leqq t \leqq T)$, then

$$
\left|\mu^{p+1} \int_{0}^{t} F(\tau) e^{\mu^{2}(\tau-t)} d \tau\right|^{2} \leqq \frac{1}{2} \int_{0}^{t}\left|\mu^{p} F(\tau)\right|^{2} d \tau
$$

where $p=0$ or 1 and $0 \leqq t \leqq T$.

We set up the successive approximations

$$
\begin{aligned}
& x_{k}{ }^{(0)}(t)=a_{k} e^{-\mu_{k}^{2} t}, \cdots, \\
& x_{k}^{(n)}(t)=a_{k} e^{-\mu_{k}^{2} t}+\int_{0}^{t} f_{k}\left\{\tau, x^{(n-1)}(\tau)\right\} e^{\mu_{k}^{2}(\tau-t)} d \tau, \cdots .
\end{aligned}
$$

Obviously $\left[x^{(0)}(t)\right]$ is in $R(r)$. Assume for the moment that $\left[x^{(n-1)}(t)\right]$ is in $R(r)$ for $0 \leqq t \leqq K$; then, if the $x_{k}^{(n-1)}(t)$ are also continuous, we have

$$
\begin{aligned}
\left|\mu^{2}\left[x^{(n)}(t)-a e^{-\mu^{2} t}\right]\right|^{2} & =\left|\mu^{2} \int_{0}^{t} f\left\{\tau, x^{(n-1)}(\tau)\right\} e^{\mu^{2}(\tau-t)} d \tau\right|^{2} \\
& \leqq \frac{1}{2} \int_{0}^{t}\left|\mu f\left\{\tau, x^{(n-1)}(\tau)\right\}\right|^{2} d \tau \\
& \leqq B^{2} t \leqq r^{2} \text { for } 0 \leqq t \leqq T, r^{2} / B^{2} .
\end{aligned}
$$

Hence we have shown by induction that $\left[x^{(n)}(t)\right]$ lies in $R(r)$ for $0 \leqq t \leqq K$, and that the $x_{k}^{(n)}(t)$ are continuous.

Also 


$$
\begin{aligned}
\left|\mu\left[x^{(1)}(t)-x^{(0)}(t)\right]\right|^{2} & =\mid \mu\left[x^{(1)}(t)-\left.a e^{-\mu^{2} t} t\right|^{2}\right. \\
& \leqq\left|\mu^{2}\left[x^{(1)}(t)-a e^{-\mu^{2} t}\right]\right|^{2} \leqq B^{2} t .
\end{aligned}
$$

Using (7.6) and Hypothesis 3, we get

$$
\begin{aligned}
\left|\mu\left[x^{(n+1)}(t)-x^{(n)}(t)\right]\right|^{2} & =\left|\mu \int_{0}^{t}\left[f\left\{\tau, x^{(n)}(\tau)\right\}-f\left\{\tau, x^{(n-1)}(\tau)\right\}\right] e^{\mu^{2}(\tau-t)} d \tau\right|^{2} \\
& \leqq \frac{1}{2} \int_{0}^{t}\left|f\left\{\tau, x^{(n)}(\tau)\right\}-f\left\{\tau, x^{(n-1)}(\tau)\right\}\right|^{2} d \tau \\
& \leqq \int_{0}^{t} A^{2} \cdot\left|\mu\left[x^{(n)}(\tau)-x^{(n-1)}(\tau)\right]\right|^{2} d \tau
\end{aligned}
$$

We thus have a recursion formula which enables us to prove by induction that

$$
\left|\mu\left[x^{(n)}(t)-x^{(n-1)}(t)\right]\right|^{2} \leqq \frac{A^{2(n-1)} B^{2} t^{n}}{n !} .
$$

By the Weierstrass test we see that the successive approximations converge uniformly: $\lim _{n=\infty} x_{k}^{(n)}(t)=x_{k}(t)$ uniformly, this relation being regarded as defining the $x_{k}(t)$ for $0 \leqq t \leqq K$.

Using (7.3) we have

$$
\left|\mu\left[x(t)-x^{(n)}(t)\right]\right| \leqq \sum_{m=n}^{\infty}\left|\mu\left[x^{(m+1)}(t)-x^{(m)}(t)\right]\right| \leqq \sum_{m=n}^{\infty}\left(\frac{A^{2 m} B^{2} K^{m+1}}{(m+1) !}\right)^{1 / 2} .
$$

Then from Hypothesis 3, we have

$$
\left|f\{t, x(t)\}-f\left\{t, x^{(n)}(t)\right\}\right| \leqq 2^{1 / 2} A \sum_{m=n}^{\infty}\left(\frac{A^{2 m} B^{2} K^{m+1}}{(m+1) !}\right)^{1 / 2} .
$$

And, since the right hand member of this inequality may be taken arbitrarily small independently of $t(0 \leqq t \leqq K)$, we have $\lim _{n=\infty} f_{k}\left\{t, x^{(n)}(t)\right\}=f_{k}\{t, x(t)\}$ uniformly. It is therefore obvious that $[x(t)]$ constitutes a solution of (7.2). It also satisfies (7.1) almost everywhere on $0 \leqq t \leqq K$.

The proof of the uniqueness of this solution follows essentially the same lines and is left to the reader.

8. Application to the partial differential equation. In applying the results of the preceding article we shall take $\mu_{k}=k ; q=$ the lesser of the two numbers $6^{1 / 2} U / \pi$ and $6^{1 / 2} P / \pi$. And we shall assume (as in $\S 7$ ) that $q-\left|a \mu^{2}\right|$ $=r>0$. We define the $f_{k}\{t, x\}$ as follows.

Let $[x]$ be an arbitrary point of $Q(q) \cdot\left|\mu^{2} x\right| \leqq q$. Then from Schwarz's inequality we have 


$$
\begin{aligned}
\sum_{k=1}^{\infty} k\left|x_{k}\right| & =\sum_{k=1}^{\infty}\left(k^{2} \cdot\left|x_{k}\right|\right)\left(\frac{1}{k}\right) \leqq\left(\sum_{k=1}^{\infty} k^{4} x_{k}^{2}\right)^{1 / 2}\left(\sum_{k=1}^{\infty} \frac{1}{k^{2}}\right)^{1 / 2} \\
& =\left|\mu^{2} x\right| \cdot \frac{\pi}{6^{1 / 2}} \leqq \frac{\pi q}{6^{1 / 2}} \leqq U \text { and } P .
\end{aligned}
$$

It follows that the two series $u^{\prime}(y)=\sum_{k=1}^{\infty} k x_{k} \cos k y$ and $u(y)=\sum_{k=1}^{\infty} x_{k}$ sin $k y$ converge uniformly. Moreover $\left|u^{\prime}(y)\right| \leqq P$ and $|u(y)| \leqq U$. By the RieszFischer theorem we also have a function $u^{\prime \prime}(y)$, defined almost everywhere on $0 \leqq y \leqq \pi$, such that its square is summable on this interval and such that

$$
(2 / \pi) \int_{0}^{\pi} u^{\prime \prime}(y) \sin k y d y=-k^{2} x_{k}
$$

and

$$
(2 / \pi) \int_{0}^{\pi}\left[u^{\prime \prime}(y)\right]^{2} d y=\left|\mu^{2} x\right|^{2} \leqq q^{2} .
$$

Integrating (8.1) by parts, it is easily seen that $u^{\prime \prime}(y)$ is really the second derivative of $u(y)$, a fact anticipated in the notation. Thus corresponding to any point $[x]$ in $Q(q)$ we can write down a function $u(y)$ with the properties enunciated above. We are now in a position to use this function to define

$$
f_{k}\{t, x\}=(2 / \pi) \int_{0}^{\pi} F\left[u^{\prime}(y), u(y), y, t\right] \sin k y d y .
$$

Integrating this by parts we have

$$
f_{k}\{t, x\}=-\left.\frac{2}{\pi} F\left[u^{\prime}(y), u(y), y, t\right] \frac{\cos k y}{k}\right|_{0} ^{\pi}+\frac{2}{\pi} \int_{0}^{\pi} \frac{d F}{d y} \frac{\cos k y}{k} d y .
$$

Hence from (6.2) or $\left(6.2^{\prime}\right)$ we get

$$
k f_{k}\{t, x\}=\frac{2}{\pi} \int_{0}^{\pi}\left[\frac{\partial F}{\partial p} u^{\prime \prime}(y)+\frac{\partial F}{\partial u} u^{\prime}(y)+\frac{\partial F}{\partial y}\right] \cos k y d y .
$$

Therefore

$$
|\mu f\{t, x\}|^{2}=\frac{2}{\pi} \int_{0}^{\pi}\left[\frac{\partial F}{\partial p} u^{\prime \prime}(y)+\frac{\partial F}{\partial u} u^{\prime}(y)+\frac{\partial F}{\partial y}\right]^{2} d y .
$$

Now the right hand side of this equality is bounded (with respect to $[x]$ ) because of two facts: I. The partial derivatives of $F$, being continuous in a closed region, are bounded. II. By Schwarz's inequality and (8.2) we know that any expression of the form $\int_{0}^{\pi} G(y) u^{\prime \prime}(y) d y$ is bounded whenever $G(y)$ 
is bounded. Hence Hypothesis 2 of the preceding article holds for the definition of $f_{k}\{t, x\}$ given in (8.3).

Now let $[x]$ and $[\bar{x}]$ be two points of $Q$, to which correspond respectively the two functions $u(y)$ and $\bar{u}(y)$. We have from (6.3)

$$
\begin{aligned}
|f\{t, x\}-f\{t, \bar{x}\}|^{2} & =\frac{2}{\pi} \int_{0}^{\pi}\left|F\left[u^{\prime}(y), u(y), y, t\right]-F\left[\bar{u}^{\prime}(y), \bar{u}(y), y, t\right]\right|^{2} d y \\
& \leqq \frac{2}{\pi} \int_{0}^{\pi}\left[2 \alpha^{2} \cdot\left|u^{\prime}(y)-\bar{u}^{\prime}(y)\right|^{2}+2 \beta^{2} \cdot|u(y)-\bar{u}(y)|^{2}\right] d y \\
& =2 \alpha^{2} \sum_{k=1}^{\infty}\left(k x_{k}-k \bar{x}_{k}\right)^{2}+2 \beta^{2} \sum_{k=1}^{\infty}\left(x_{k}-\bar{x}_{k}\right)^{2} \\
& \leqq 2\left(\alpha^{2}+\beta^{2}\right) \cdot|\mu(x-\bar{x})|^{2} .
\end{aligned}
$$

Hence Hypothesis 3 holds with $A=\left(\alpha^{2}+\beta^{2}\right)^{1 / 2}$.

The proof that Hypothesis 1 holds is easy and is left to the reader.

Hence all the results of $\S 7$ are now available. Using the $x_{k}(t)$, the existence of which was asserted in that article, we form the function $u(y, t)=\sum_{k=1}^{\infty}$ $x_{k}(t) \sin k y$. Then $u(y, t)$ satisfies (6.1) almost everywhere in the region $0 \leqq y \leqq \pi$, $0 \leqq t \leqq K$, and also fulfills the boundary conditions (6.4). Furthermore $u(y, t)$ is the only function of class $C^{\prime}$ in $y$ and possessing derivatives $\partial u / \partial t, \partial^{2} u / \partial y^{2}$ almost everywhere, such that $|u(y, t)| \leqq U,|\partial u / \partial y| \leqq P$, which enjoys these properties.

In the first place by (7.1), (7.4), and Hypothesis 2, it is seen that $\sum_{k=1}^{\infty}$ $\left[d x_{k} / d t\right]^{2}$ converges. By the Riesz-Fischer theorem we can therefore find a function $w(y, t)$ whose Fourier coefficients are precisely these $d x_{k} / d t$. It is now an elementary exercise in analysis to identify $w(y, t)$ with $\partial u / \partial t$.

The theorem follows from the fact that the Fourier coefficients of

$$
\frac{\partial u}{\partial t}-\frac{\partial^{2} u}{\partial y^{2}}-F\left(\frac{\partial u}{\partial y}, u, y, t\right)
$$

are none other than the

$$
\frac{d x_{k}}{d t}+k^{2} x_{k}-f_{k}\{t, x\}
$$

all of which vanish. The uniqueness part of the theorem follows from the fact that the assumption of the existence of a second such function, $\tilde{u}(y, t)$, leads to a contradiction of the uniqueness of the functions $[x(t)]$.

Cambridge, Mass. 